\title{
Konevets Island (Leningrad Region, Russia) - a historical refuge of lichen diversity in Lake Ladoga
}

\section{Dmitry E. Himelbrant ${ }^{1,2}$, Irina S. Stepanchikova ${ }^{1,2}$, Ekaterina S. Kuznetsova ${ }^{1,2}$, Jurga Motiejūnaité ${ }^{3}$, Ludmila A. Konoreva ${ }^{2,4}$}

\author{
${ }^{1}$ St. Petersburg State University, Universitetskaya emb. 7-9, 199034 St. Petersburg, Russia. \\ E-mails: d_brant@mail.ru, stepa_ir@mail.ru, igel_kuzn@mail.ru \\ ${ }^{2}$ Laboratory of Lichenology and Bryology, Komarov Botanical Institute RAS, Professor Popov St. 2, \\ 197376 St. Petersburg, Russia \\ ${ }^{3}$ Laboratory of Mycology, Institute of Botany, Nature Research Centre, Žaliųjų Ežerų 49, LT-08406 Vilnius, Lithuania. \\ E-mail: jurga.motiejunaite@botanika.lt \\ ${ }^{4}$ Polar-alpine Botanical Garden-Institute, 184250 Murmansk Region, Kirovsk. E-mail: ajdarzapov@yandex.ru
}

\begin{abstract}
We present an updated checklist for Konevets Island (Leningrad Region, Russia). The revealed lichen biota comprises 435 species, including 378 lichens, 46 lichenicolous fungi and 11 non-lichenized saprobic fungi, of which 31 species (27 lichens and 4 lichenicolous fungi) are known only from collections made by Veli Räsänen (1917, 1938). Acremonium hypholomatis is reported for the first time for Russia; Caloplaca soralifera, Trapelia corticola, and Muellerella lichenicola for Northwestern European Russia; and Bacidia vermifera, Lecanora mughicola, Micarea contexta, Pyrenochaeta xanthoriae, Rhizocarpon disporum, Stigmidium squamariae and Xylographa difformis for Leningrad Region. From lichenological point of view, the most valuable habitats of Konevets Island are old-growth spruce forests. The studied lichen biota is rich and diverse and exceptionally wellpreserved in comparison to the mainland part of Karelian Isthmus. It definitely deserves protection.
\end{abstract}

Keywords: Lake Ladoga, Isthmus karelicus, Räsänen, old-growth forests, Acremonium hypholomatis, Caloplaca soralifera, Trapelia corticola, Muellerella lichenicola

\section{INTRODUCTION}

Konevets Island (Konevitsa in Finnish) is the only large island in Lake Ladoga within the boundaries of Leningrad Region. An outstanding Finnish lichenologist Veli Räsänen (VR) collected lichens there during two short trips on August 12, 1917 and June 15-17, 1938. His work in 1938 was carried out with the blessing of Hegumen Mavriky, head of the Konevsky Monastery. During these four days Räsänen investigated the vegetation and collected lichen samples very intensively - about 290 specimens are kept in the Botanical Museum, Finnish Museum of Natural History, University of Helsinki (H), 6 specimens have also been found in the Museum of Evolution, Uppsala University (UPS). The main results of this work listing 114 lichen species (in modern understanding) were published (Räsänen, 1944), several species were also mentioned in earlier publications (Räsänen, 1921, 1939a, b, 1940). Three more species were published by other authors, who had examined Räsänen's collections (Ahti \& Hyvönen, 1985; Halonen et al., 1999; Kukwa, 2011). Some of the records published for Konevets by Räsänen were cited later in different monographs and papers (Magnusson, 1947; Ahlner, 1948; Hakulinen, 1949, 1962; Tibell, 1973; Ekman, 1997).

Dmitry Himelbrant (DH), Ekaterina Kuznetsova (EK) and Irina Stepanchikova (IS) performed a critical revision of Räsänen's collection in H, with much appreciated help of Teuvo Ahti, in 2007-2009 and 2012, and a revision of the collection in UPS in 2015. As a result, the identification of several species was revised, additional species were discovered, and a total of 178 species collected by Räsänen were accepted for the present list of lichen flora of Konevets Island. Four species were recently published as new for Leningrad Region (Himelbrant et al., 2016). The same three authors conducted a comprehensive field study of Konevets lichens in 2017, with the blessing of Hegumen Alexander, head of the Konevsky Monastery. The aim of this paper is to present all known data on lichens and allied fungi of Konevets Island. 


\section{STUDY AREA}

Konevets Island belongs administratively to Priozersk District and biogeographically to Isthmus karelicus, a province of the Eastern Fennoscandia (Kotiranta et al., 1998). It lies in the western part of the great Lake Ladoga, $3.5 \mathrm{~km}$ from the shore, and occupies an area of c. $9.5 \mathrm{~km}^{2}$ (including 10 very small in-shore islets). Konevets is the only island in this part of the lake, in contrast to the northern part of Ladoga, where a number of rocky islands are present. The relief of the island is more or less uniform - most part of the territory presents rather low flatland (average height is $3 \mathrm{~m}$ a.s.1.) with two central elevations (Svyataya and Zmeinaya hills) of about $30 \mathrm{~m}$ a.s.1. The whole island is composed of sedimentary (lacustrine) and moraine sand with granite moraine boulders of various size; rocky outcrops are absent. The island is bordered by fine-sand shores, abrupt in the west and sloping in the east. Considerable part of Konevets is covered by pine and spruce forests; small-leaved forests are also present in disturbed places and some shores.

Konevsky Monastery was founded on the island at the end of the 14th century and, with some interruptions in the 16 th, 17 th and 20 th centuries, exists till the present. Most of modern monastery buildings were erected in the 19th century. The history of Konevets Island in the 20th century is complicated. From 1918 to 1940 it belonged to Finland (Finnish parish Pyhäjärvi), and during the World War II in 1941-1944 the island was occupied by the Finnish army most of the time. After the war Konevets became a territory of the Soviet Union military base and was closed for visitors until 1991, when the Monastery was re-established (Konevets..., 2015). Civil or laity inhabitants, settlements and industry have been absent from the island throughout its history, but anthropogenic activity from the Monastery and military base has affected the nature of Konevets: monastery buildings, piers, meadows, gardens, alleys of broadleaved trees, plantations of introduced plants, as well as clear cuttings are found in the southern part of the island, while roads, wastelands and ruins of military constructions are present throughout the area. Nevertheless, the semi-closed regime of the island provided better preservation of natural communities, but the biodiversity of the island is considerably less studied compared to the mainland.

\section{MATERIAL AND METHODS}

The fresh material was collected on Konevets Island from July 26 to August 5, 2017 by DH, EK and IS. Altogether we investigated 65 localities (Appendix 1; Fig. 1): 34 standard $20 \times 20 \mathrm{~m}$ sample areas (or in natural boundaries of the community), where the lichen diversity on each substrate was described as detailed as possible, and 31 additional plots, where only individual substrates and species were recorded. All geographical coordinates are given in coordinate system WGS 1984. The specimens are deposited in the herbaria of the Botanical Museum, University of Helsinki (H), Department of Botany, St. Petersburg State University (LECB) and Institute of Botany, Nature Research Centre in Vilnius (BILAS). Additionally, we included four exact points, mentioned by Räsänen in his publications or some herbarium labels, in the list of localities. Most Räsänen's records, however, are indicated as 'Konevitsa' without further details; in this case the locality is not indicated in the species list. DH, IS and EK identified the majority of specimens, Jurga Motiejūnaite performed identification of most of the lichenicolous fungi, and Ludmila Konoreva identified most of the Micarea specimens; if otherwise, the author of identification is indicated.

In the species list the nomenclature of taxa generally follows Nordin et al. (2011), except for the names Agyrium rufum, Lepra spp., Usnea diplotypus (see Hafellner \& Türk, 2016), Pachyphiale fagicola (Gagarina, 2015), Porpidia macrocarpa f. nigrocruenta (Fryday, 2005) and Phaeocalicium polyporaeum (Titov, 2006). For each species the substrates and localities are listed. Species new to Leningrad Region or larger regions are accompanied by information on diagnostic characteristics and distribution in Northwestern European Russia, Fennoscandia and the Baltic countries. Lichen substances are given for TLC-analyzed species. Chromatography was performed by IS, DH (different taxa), Ludmila Konoreva and Sergei Chesnokov (Micarea spp.) according to the standard techniques of high performance thin-layer chromatography using solvent systems A, B and C (Orange et al., 2001). Lichenicolous and algicolous fungi and protists are marked with \#, non-lichenized fungi with +, habitat specialists with s, and indicator species with ${ }^{\text {i }}$ (see Andersson et al., 2009); regions are abbreviated as follows: LR - Leningrad Region, 


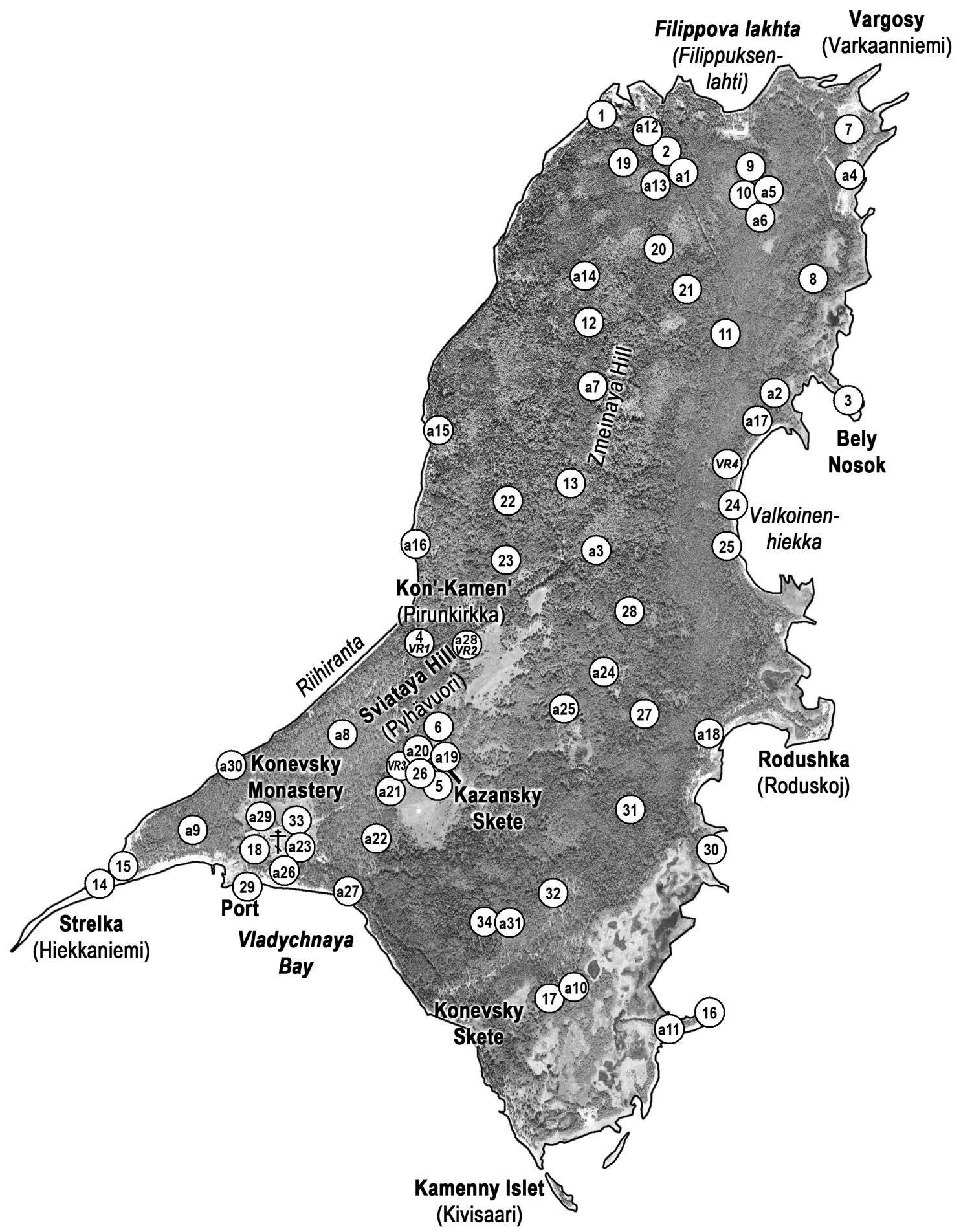

Fig. 1. The study area, Konevets Island (Konevitsa), with location of collection sites. 
ELR - Eastern Leningrad Region, WLR - Western Leningrad Region, $\mathrm{SPb}$ - St. Petersburg. For each species collected in 2017 the frequency of occurrence is indicated in square brackets: single record $\left[R_{1}\right]$ - the species was found once, rare $[R]$ - recorded in 2-6 standard sample areas or more than in one additional plot, occasionally $[\mathrm{O}]-7-13$, frequent $[\mathrm{F}]-14-20$, common $[\mathrm{C}]$ - 21-27, very common [VC] - 28-34 standard sample areas.

\section{THE SPECIES}

\# Abrothallus bertianus De Not. - coll. VR: on thallus of Melanohalea olivacea on bark of Alnus incana (L.) Moench, 16.06.1938 (H 6022473: det. Teuvo Ahti, 2008).

\# Abrothallus CETRARIAe I. Kotte - on thallus of Platismatia glauca on bark of Picea abies (L.) H. Karst. branch; $20\left[\mathrm{R}_{1}\right]$.

\# ABRothallus PaRmeliarum (Sommerf.) Arnold on thalli of Cetraria islandica on sandy soil; 24, 32, a16 [R].

Absconditella lignicola Vězda $\&$ Pišút - on wood of conifers, decaying fruit bodies of polypores; 8, 9, 19, 21, 28 [R].

ACAROSPORA FUSCATA (Schrad.) Th. Fr. - on granite boulders and pier; 1, 14, 29, 30, a16, a25 [R].

ACAROSPORA MOENIUM (Vain.) Räsänen - on concrete; $3\left[R_{1}\right]$.

ACAROSPORA VERONEnsis A. Massal. - on granite boulder, concrete, slate, iron; 3, 29, 30 [R].

\# ACREMONIUM HYPHOLOMATIS (Boedijn) D. Hawksw. - on thalli and apothecia of epiphytic Physcia spp.; 6, 33 [R]. - New to Russia. Distribution in Fennoscandia and Baltic countries: not reported. Described from Indonesia and New Guinea; first lichenicolous record refers to Germany (Diederich \& Braun, 2009). Facultatively lichenicolous hyphomycetous fungus, characterized by formation of pale pinkish colonies on host lichen thallus. Superficially the colonies are reminiscent of those of Illosporiopsis christiansenii (B. L. Brady \& D. Hawksw.) D. Hawksw., though the colour of the latter is brighter pink. Microscopically, however, A. hypholomatis differs significantly by its erect conidiophores and ellipsoid, apically rounded, basally narrowly truncate conidia. Of all known Acremonium species, A. hypholomatis is distinguished by large, $11.5-20 \times 5.5-7 \mu \mathrm{m}$ conidia (Diederich \& Braun, 2009). Meas- urements of conidia in our specimen were concurrent with those given by Diederich \& Braun (2009).

Acrocordia CAVATA (Ach.) R. C. Harris - on bark of Populus tremula L.; $34\left[\mathrm{R}_{1}\right]$.

+ AgYrium Rufum (Pers.) Fr. - on wood of Picea abies branches; $9\left[\mathrm{R}_{1}\right]$.

${ }^{i}$ Alectoria sarmentosa (Ach.) Ach. subsp. SARMENTOSA - on bark of Picea abies; a1, a2 [R]. Red Data Book of LR (Tzvelev, 2000).

Alyxoria CUlmigena (Libert) Ertz - coll. VR: on bark of Acer platanoides L., 15.06.1938 (Himelbrant et al., 2017; H 8005159, sub Lecanora argentata).

AlYXORIA VARIA (Pers.) Ertz \& Tehler - on bark of Acer platanoides, Fraxinus excelsior L., Malus domestica Borkh., Populus balsamifera, Quercus robur L.; 4, 18, 26, a20, a26 [R]. Coll. VR: on bark of Acer platanoides, 12.08.1917, 15.06.1938 (Räsänen, 1944; H).

Amandinea Punctata (Hoffm.) Coppins \& Scheid. - on bark of Acer platanoides, Fraxinus excelsior, Populus balsamifera, Quercus robur, Tilia cordata Mill., wood; 5, 18, 29, a19, a20, a26 [R]. Coll. VR: on bark of Acer platanoides and Tilia sp., on lignum, 15-16.06.1938 (Räsänen, 1944; H).

ANAPTYCHIA CILIARIS (L.) Körb. - on bark of Acer platanoides, Populus tremula; 18, a15, a19 [R]. Coll. VR: on bark of Acer platanoides, 15.06.1938 (Räsänen, 1944; Hakulinen, 1962; H 8003913).

ANISOMERIDIUM POLYPORI (Ellis \& Everh.) M. E. Barr - on bark of Populus balsamifera, Quercus robur, $18\left[\mathrm{R}_{1}\right]$.

ARCTOPARMElia CEnTRIFuga (L.) Hale - on granite boulder; $32\left[\mathrm{R}_{1}\right]$. Red Data Book of LR (Tzvelev, 2000).

ARthonia APATEtica (A. Massal.) Th. Fr. - on bark of Fraxinus excelsior, 4 [ $\left.\mathrm{R}_{1}\right]$.

ARTHONIA DIDYMA Körb. - on bark of Fraxinus excelsior, Populus tremula; 2, 4 [R]. Coll. VR: on bark of Tilia cordata, 15.06.1938 (H 8004022, sub Physcia tenella).

ARTHONIA DISPUNCTA Nyl. - on bark of Acer platanoides; $4,5[\mathrm{R}]$.

ARTHONIA FUSCA (A. Massal.) Hepp - on concrete; $3\left[R_{1}\right]$.

ARTHONia MEdiella Nyl. - on bark of Acer platanoides, Fraxinus excelsior, Picea abies, Quercus robur, Sorbus aucuparia L.; 2, 4, 5, 8, 12, 13, 20, 25, 27, a26 [O]. Reported by VR (Räsänen, 1944), but the specimen refers to Bacidia igniarii. 
ARthonia Patellulata Nyl. - on bark of Populus tremula; 6, 16, 17, 34 [R].

ARTHONIA PUNCTIFORMIS Ach. - on bark of Acer platanoides, Betula spp., Malus domestica, Populus tremula, Quercus robur, Salix spp.; 4, 5, 16, 20, 26, a15 [R].

ARthonia Radiata (Pers.) Ach. - on bark of Populus balsamifera, P. tremula, Sorbus aucuparia; 4, 18, 34 [R]. Coll. VR: VR2 and without distinct locality, on bark of Fraxinus excelsior, Quercus robur, Salix spp., Tilia cordata, 15-16.06.1938 (Räsänen, 1944; H).

ARTHONIA RUANA A. Massal. - on bark of Acer platanoides, Sorbus aucuparia; 4, 34 [R].

i ARTHONIA SPADICEA Leight. - on bark of Acer platanoides, Populus tremula, bark and wood (log) of Picea abies; 5, 9, 28, a15 [R].

i ARTHONIA VINOSA Leight. - on bark of Populus tremula, Quercus robur, 5, 34, a19 [R]. Coll. VR: on bark of Picea abies, 15.06.1938 (H 8004410, sub Chrysothrix candelaris).

ARTHOPYRENIA ANALEPTA (Ach.) A. Massal. - on bark of Sorbus aucuparia; $27\left[\mathrm{R}_{1}\right]$.

+ ARTHothelium SCANDINAVICUM Th. Fr. - on bark of Picea abies; 9, 12, 13, 27, 31 [R]. Coll. VR: on bark of Picea abies, 15.06.1938 (Räsänen, 1944; H 8004065).

ARTHROSPORUM POPULORUM A. Massal. - on bark of Populus tremula, Siringa vulgaris L.; 16, 33, a15 [R].

AsPiCILIA CINEREA (L.) Körb. - on granite boulders and pier; 1, 29, 30, a25 [R]. Coll. VR: on granite, 16-17.06.1938 (Räsänen, 1944; H).

ASPICILIA KARELICA (H. Magn.) Oxner - coll. VR: on granite boulders on the shore, 16.06.1938 $(\mathrm{H}$ s. n.). - New to WLR, previously known from ELR (Kuznetsova et al., 2007).

Aspicilia LAEVATA (Ach.) Arnold - on granite boulders and pier; $1,29[R]$.

ASPICILIA VERRUCIGERA Hue - on granite pebbles; a16 $\left[R_{1}\right]$.

AtHallia CERINella (Nyl.) Arup, Frödén \& Søchting - coll. VR: on bark of Acer platanoides, 15.06.1938 (H 8003941).

Athallia holocarpa (Hoffm.) Arup, Frödén \& Søchting - on concrete, iron; 3 [ $\left.R_{1}\right]$.

Athallia PyRACEa (Ach.) Arup, Frödén \& Søchting - on bark of Acer platanoides, Malus domestica, Populus tremula, Siringa vulgaris, Sorbus aucuparia, wood, concrete; 3, 5, 6, 16, 17, 26, 29, 33, 34, a15 [O]. Coll. VR: on bark of Acer platanoides, 15.06.1938 (H 8003941).

\# ATHELIA ARACHNOIDEA (Berk.) Jülich - on algae on bark of Siringa vulgaris; $33\left[\mathrm{R}_{1}\right]$.
BACIDIA ARCEutina (Ach.) Arnold - on bark of Acer platanoides, Populus tremula; 21, 27 [R]. Coll. VR: on rotten wood, 16.06.1938 (H 8003504).

BACIDIA CIRCUMSPECTA (Nyl. ex Vain.) Malme - on bark of Populus tremula; 17 [ $\left.\mathrm{R}_{1}\right]$.

${ }^{i}$ BACIDIA FRAXINEA Lönnr. - on bark of Acer platanoides, Populus tremula; 4, 18, 21, 22, 34, a14 [R]. - New to WLR, previously known from ELR (Kuznetsova et al., 2007).

BACIDIA IGNIARII (Nyl.) Oxner - on bark of Populus tremula; $6\left[\mathrm{R}_{1}\right]$. Coll. VR: on wood of Pinus sylvestris L., 16.06.1938 (H 8004028).

BACIDIA LAUROCERASI (Delise ex Duby) Zahlbr. - on bark of Sorbus aucuparia; 27 [ $\left.\mathrm{R}_{1}\right]$. Coll. VR: on bark of Sorbus aucuparia, Tilia cordata, 16.06.1938 (Räsänen, 1944; H 8004097, 8004099).

i Bacidia POlYChroa (Th. Fr.) Körb. - on bark of Populus tremula; 21, 34 [R].

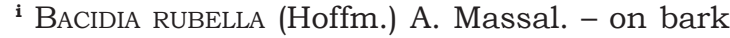
of Acer platanoides, Populus balsamifera; $18,21[\mathrm{R}]$.

BACIDIA SUBINCOMPTA (Nyl.) Arnold - on bark of Acer platanoides, Populus tremula, Quercus robur, 4, 6, 12, 18, 21, 22, 34, a19, a23 [O].

BACIDIA VERMIFERA (Nyl.) Th. Fr. - on wood; 5 [R ${ }_{1}$. - New to LR. Previously reported from SPb (Rassadina, 1930), but the specimen belongs to $B$. arceutina. Distribution in Northwestern European Russia outside of LR: Republic of Karelia (Fadeeva et al., 2007). Distribution in Fennoscandia and Baltic countries: Norway, Sweden, Finland (Nordin et al., 2011), Estonia (Randlane et al., 2016), Latvia (Ābolina et al., 2015). Characterized by black apothecia with reddish, $\mathrm{K}+$ intensifying purple exciple and epihymenium and worm-like or shortacicular spores (Smith et al., 2009).

BACIDINA CHLOROTicula (Nyl.) Vězda \& Poelt - on plant debris, iron; a10, a27 [R].

BACIDINA INUNDATA (Fr.) Vězda - on wet wood of old pier; $29\left[\mathrm{R}_{1}\right]$. Coll. VR: on same substrate, 16.06.1938 (Räsänen, 1944; H 8004112, 8004113).

BAEOMYCES CARNEUS Flörke - on soil, large granite boulder (vertical shaded and wet surface); 23, a30 [R].

BAeOMyces Rufus (Huds.) Rebent. - on granite boulder Kon'-Kamen'; 4 [R $\left.{ }_{1}\right]$.

Biatora albohyalina (Nyl.) Bagl. \& Carestia - on bark of Sorbus aucuparia; 27 [ $\left.\mathrm{R}_{1}\right]$.

BIATORA BeCKHAUSil (Körb.) Tuck. - coll. VR: on timber of Pinus sylvestris (wooden pier), 16.06.1938 (Räsänen, 1944; H 8004089). 
BiATORA EFFLORESCENS (Hedl.) Räsänen - on bark of Acer platanoides, Alnus incana, Betula spp., Fraxinus excelsior, Picea abies, Populus tremula, Quercus robur, Tilia cordata; 1 , $2,4-6,8,12,18,21-23,27,31,34$, a15, a26 [F].

Biatora GLOBUlosa (Flörke) Fr. - on bark of Acer platanoides, Malus domestica, Populus tremula, Quercus robur, Tilia cordata; 2, 18, 26, a26 [R]. Coll. VR: on bark of Quercus robur, 15.06.1938 (Räsänen, 1944; H 8004093).

Biatora HELVOLA Körb. ex Hellb. - on bark of Acer platanoides, Picea abies, Populus tremula, Sorbus aucuparia; 2, 4, 21, 27, 31, 34 [R]. Coll. VR: on bark of Quercus robur and wood of Pinus sylvestris, 15-16.06.1938 (H 8004093, 8004099).

BIATORA OCELLIFORMIS (Nyl.) Arnold - on bark of Acer platanoides, Sorbus aucuparia; 4, 27 [R]. Coll. VR: on bark of Quercus robur, wood of Pinus sylvestris, 15-16.06.1938 (H 8004093, 8004099).

Biatora sphaeroidiza (Vain.) Printzen \& Holien - on bark of Acer platanoides, Picea abies; $2,4[R]$.

s BiATORIDIUM MONASTERIENSE J. Lahm ex Körb. - on bark of Acer platanoides; $21\left[\mathrm{R}_{1}\right]$.

\# Biatoropsis usNeARum Räsänen - on thallus of Usnea hirta on bark of Pinus sylvestris; $19\left[\mathrm{R}_{1}\right]$. Coll. VR: on thallus of Usnea subfloridana on bark of Betula sp., 1938? (H 8003694).

Bilimbia MicrocarPa (Th. Fr.) Th. Fr. - on mosses on bark of Populus tremula; 22, 27 [R].

BRIANARIA SYLVICOLA (Flot. ex Körb.) S. Ekman \& M. Svensson - on iron; a9 $\left[R_{1}\right]$.

\# BRIANCOPPINSIA CYTOSPORA (Vouaux) Diederich, Ertz, Lawrey \& van den Boom - on thallus of Hypogymnia physodes on bark of Picea abies, on thallus of Parmeliopsis ambigua on bark of Betula sp.; 8, 19 [R].

BRYORIA CAPILlARIS (Ach.) Brodo \& D. Hawksw. - on bark of Betula spp., Picea abies, wood of conifers; 2, 8, 10,12, 13, 20, 22, 23, 25, 27, 28, 31, 32 [O]. Coll. VR: VR4 and without distinct locality, on bark of Acer platanoides, Betula spp., Picea abies, Pinus sylvestris, wood of conifers and granite boulder, 12.08.1917, 1938 (Räsänen, 1944; H).

BRYORIA FURCELLATA (Fr.) Brodo \& D. Hawksw. - on bark of Betula spp., Fraxinus excelsior, Picea abies, Pinus sylvestris, wood of conifers; 5, 10, 13, 19, 20, 23, 31, 32, a1, a2, a29 [O].

BRYORIA FUSCESCENS (Gyeln.) Brodo \& D. Hawksw. - on bark of Acer platanoides, Betula spp.,
Fraxinus excelsior, Malus domestica, Picea abies, Pinus mugo, P. sylvestris, Populus balsamifera, Quercus robur, Tilia cordata, wood of conifers, worked timber and sandy soil; 2 , 4, 5, 8-10, 12-15, 18, 19, 21, 23-27, 29, 31, 32, a29 [C]. Coll. VR: on bark of Betula spp. and Picea abies, on wood, 15-16.06.1938 (H).

BRYORIA IMPLEXA (Hoffm.) Brodo \& D. Hawksw. s. 1. - on bark of Picea abies; $13\left[\mathrm{R}_{1}\right]$. Thallus contains norstictic and connorstictic acids. Reported by VR (Räsänen, 1944), but the specimens refer to $B$. capillaris.

BRYORIA NADVORNIKIANA (Gyeln.) Brodo \& D. Hawksw. - on bark of Picea abies; 23 [ $\mathrm{R}_{1}$ ]. Red Data Book of LR (Tzvelev, 2000).

BuElLIA ARBOREA Coppins \& Tønsberg - coll. VR: on wood, 16.06.1938 (Himelbrant et al., 2016; H 8005508, sub Imshaugia aleurites).

s BuELLIA ARNOLDII Servit - on bark of Betula sp.; $19\left[R_{1}\right]$.

Buellia DISCIFORMIS (Fr.) Mudd - on bark of Acer platanoides, Alnus incana, Populus tremula, Quercus robur, 1, 5, 17, 18, a19 [R]. Coll. VR: on bark of Acer platanoides, Alnus incana, Quercus robur, Salix spp., 15-16.06.1938 (Räsänen, 1944; H).

Buellia eRUBESCEns Arnold - on bark of Acer platanoides, Populus tremula; 4, 17 [R]. Coll. VR: VR2 and without distinct locality, on bark of Alnus incana, Sorbus aucuparia, Tilia cordata, 15-16.06.1938 (Räsänen, 1944; H).

BuElLIA GRISEOviRENS (Turner \& Borrer ex Sm.) Almb. - on wood; 9, 29 [R].

Buellia SCHAERERI De Not. - on bark of Quercus robur; $5\left[\mathrm{R}_{1}\right]$. Coll. VR: VR3, on wood, 15.06.1938 (Räsänen, 1944; H 8004245).

i Calicium Denigratum (Vain.) Tibell - on standing deadwood of Pinus sylvestris; a6 $\left[\mathrm{R}_{1}\right]$.

Calicium glaucellum Ach. - on bark of Acer platanoides, Pinus sylvestris, wood; 5, 10, 11, $18,19,23,26$, a26 [O].

Calicium salicinum Pers. - on bark of Malus domestica, Quercus robur, lignum of Acer platanoides, Malus domestica; 5, 18, 26, a26 [R].

CAlicium trabinellum (Ach.) Ach. - on standing deadwood of Pinus sylvestris; 10 [ $\left.\mathrm{R}_{1}\right]$.

CAlicium VIRIDE Pers. - on bark of Abies sibirica Ledeb., Picea abies, Quercus robur, 5, 18, $25[\mathrm{R}]$.

Caloplaca cerina (Hedw.) Th. Fr. - on bark of Malus domestica, Populus tremula; 17, 26, a15 [R]. Coll. VR: on bark of Sorbus aucuparia, 15.06.1938 (Räsänen, 1944; H 8005149). 
Caloplaca obscurella (J. Lahm ex Körb.) Th. Fr. - on bark of Malus domestica, Sorbus aucuparia; 5, 26 [R].

CALOPLACA SORALIFERA Vondrák \& Hrouzek s. 1. on granite boulder; $16\left[\mathrm{R}_{1}\right]$. Det. Ivan Frolov, 2018. - New to North-Western European Russia. Nearest locality in European Russia known from Lipetsk Region (Muchnik et al., 2014). Distribution in Fennoscandia and Baltic countries: Norway, Sweden, Finland (Nordin et al., 2011), Lithuania (Motiejūnaite, 2017). Characterized by grey thallus consisting mostly of areoles bearing marginal granular soredia and dark orange-brown apothecia with yellowish margin (Vondrák \& Hrouzek, 2006). Similar to C. chlorina (Flot.) H. Olivier when sterile (Smith et al., 2009).

CANDElariella aurella (Hoffm.) Zahlbr. - on concrete; $3\left[R_{1}\right]$.

Candelariella coralliza (Nyl.) H. Magn. - on granite pier; $29\left[R_{1}\right]$.

Candelariella efFlorescens R. C. Harris \& W. R. Buck - on bark of Acer platanoides, Fraxinus excelsior, Malus domestica, Quercus robur, Siringa vulgaris, Tilia cordata; 5, 18, 26, 33 [R]. Coll. VR: on bark of Acer platanoides and on wood, 15-16.06.1938 (H 8000667, sub Pertusaria albescens and $\mathrm{H} 8003504$, sub Rinodina conradii).

CANDELARIELLA REFLEXA (Nyl.) Lettau - on bark of Malus domestica; $26\left[\mathrm{R}_{1}\right]$.

Candelariella vitellina (Hoffm.) Müll. Arg. - on wood, granite boulders and granite pier, slate, iron; 1, 3, 16, 29, 30 [R]. Coll. VR: on granite and timber, 16.06.1938 (Räsänen, 1944; H).

CANDELARIELla Xanthostigma (Ach.) Lettau - on bark of Acer platanoides, Malus domestica, Quercus robur, Sorbus aucuparia, Tilia cordata; 5, 18, 26 [R].

\# CARbonea supersparsa (Nyl.) Hertel - coll. VR: on thallus of Lecanora polytropa on siliceous stone in littoral zone, 16.06.1938 (Himelbrant et al., 2016; H)

\# CARBONEA VITELLINARIA (Nyl.) Hertel - coll. VR: on thallus of Candelariella vitellina on siliceous stone in littoral zone, 16.06.1938 (Himelbrant et al., 2016; H)

i Carbonicola anthracophila (Nyl.) Bendiksby \& Timdal - on standing deadwood of Pinus sylvestris; $10\left[\mathrm{R}_{1}\right]$.

CATILlaria NigRoclavata (Nyl.) Schuler - on bark of Acer platanoides; 18 [ $\left.\mathrm{R}_{1}\right]$.

CATINARIA ATROPURPUREA (Schaer.) Vězda \& Poelt - on bark and wood of Malus domestica; $26\left[\mathrm{R}_{1}\right]$.
Cetraria ericetorum Opiz subsp. ericetorum - on sandy soil; a16, a30 [R].

Cetraria islandica (L.) Ach. subsp. ISLANDiCA - on sandy soil; 3, 7, 24, 32, a16. Coll. VR: on mossy soil and wood, 16.06.1938 (Räsänen, 1944; H 8004368, 8004369). Subsp. IsLANDICA "f. SOREDIATA (Schaer.) Arnold" - on sandy soil; a30. [R].

Cetraria SePincola (Ehrh.) Ach. - on bark of Acer platanoides, Betula spp., Malus domestica, Pinus mugo, $P$. sylvestris, Populus balsamifera, $P$. tremula, on wood; $1,3,15,17,21$, 26, 27, 29, a29 [O].

${ }^{i}$ CHAENOTHECA BRACHYPODA (Ach.) Tibell - on bark of Populus tremula, on wood; 9, 12, 21, 28, 34 [R].

s Chaenotheca Chlorella (Ach.) Müll. Arg. on standing deadwood of Picea abies; 12 $\left[R_{1}\right]$. Coll. VR: VR3, on wood, 15.06.1938 (Räsänen, 1944; Tibell, 1973; H 8004397, sub Chaenotheca trichialis).

CHAENOTHECA CHRYSOCEPHALA (Turner ex Ach.) Th. Fr. - on bark of Alnus glutinosa (L.) Gaertn., Picea abies, Pinus sylvestris, Quercus robur, Tilia cordata; 2, 5, 8-10, 12, 13, 18-22, 25, 27, 28, 31, 34, a26 [F]. Coll. VR: on bark of Picea abies, 15-16.06.1938 (H 8004905, 8004906, sub Cliostomum griffithii; H s. n., sub Chrysothrix candelaris).

Chaenotheca ferruginea (Turner ex Sm.) Mig. on bark of Alnus glutinosa, Betula spp., Picea abies, Pinus sylvestris, Quercus robur, wood of conifers; 2, 5, 8-13, 19-23, 25, 27, 28, 31, 32 [F]. Coll. VR: on bark of Pinus sylvestris (H s. n., sub Hypocenomyce scalaris).

ChaEnotheca FuRfuracea (L.) Tibell - on bark of Betula spp., Picea abies, Populus tremula, standing deadwood of Picea abies; 2, 4, 21-23, 27, 34 [O].

i Chaenotheca stemonea (Ach.) Müll. Arg. - on bark of Betula spp., bark and standing deadwood of Picea abies; 2, 8, 12, 13, 20, $27,28,31$ [O].

ChaEnotheca tRICHIALIS (Ach.) Th. Fr. - on bark of Betula spp., Picea abies, Quercus robur, wood; 2, 5, 9, 12, 19-21, 27 [O]. Coll. VR: VR3, on wood, 15.06.1938 (Räsänen, 1944; H 8004393, 8004397, 8004398).

i\# Chaenothecopsis consociata (Nádv.) A. F. W. Schmidt - on bark of Picea abies, thalli of Chaenotheca chrysocephala on bark of Picea abies; 8, 12, 27, 28, 31 [R].

Chaenothecopsis Debilis (Sm.) Tibell - on bark of Quercus robur, wood of Acer platanoides; a26 $\left[R_{1}\right]$. 
i\# Chaenothecopsis nigRa Tibell - on algae on upturned roots of Picea abies; $2\left[\mathrm{R}_{1}\right]$.

\# ChaEnothecopsis pusilla (Ach.) A. F. W. Schmidt - on wood of Tilia cordata and algae on bark of Alnus glutinosa; 5, 28, a30 [R].

\# ChaEnothecopsis savonica (Räsänen) Tibell - on algae on wood of Picea abies; $2\left[\mathrm{R}_{1}\right]$.

s\# Chaenothecopsis viridireagens (Nádv.) A. F. W. Schmidt - on bark and wood of Picea abies, on thallus of Chaenotheca stemonea on bark of P. abies; 20, 28 [R]. - New to WLR, previously known from ELR (Kuznetsova et al., 2007).

CHRYSOTHRIX CANDELARIS (L.) J. R. Laundon - on bark of Fraxinus excelsior, $4\left[\mathrm{R}_{1}\right]$. Coll. VR: VR4 and without distinct locality, on bark $P i-$ cea abies, 15-16.06.1938 (Räsänen, 1939a; H 8004409, 8004410, s. n.).

CHRYSOTHRIX CHLORINA (Ach.) J. R. Laundon - on granite boulder Kon'-Kamen'; 4 [ $\left.\mathrm{R}_{1}\right]$.

Cladonia amaurocraea (Flörke) Schaer. - coll. VR: on mossy soil over boulder, 17.06.1938 (Räsänen, 1944; H 8004482).

Cladonia ARBUscula (Wallr.) Flot. subsp. ARBUSCULA - on soil, bark of Pinus mugo; 3, 7, 15, 19, 32, a11, a18. Coll. VR: on wood, 16.06.1938 (Räsänen, 1944; H 8004422); subsp. MITIS (Sandst.) Ruoss - on sandy soil; $7,14,15$, 24. [O]. Coll. VR: on wood and mossy soil, 16-17.06.1938 (Räsänen, 1944; H 8004422, 8004433, 8004434).

Cladonia BACILLIFORMis (Nyl.) Glück - on soil, bark of Pinus mugo, P. sylvestris, wood of Quercus robur, 5, 15, 19, 24 [R]. Coll. VR: on wood, 12.08.1917 (H 8004488).

Cladonia botrytes (K. G. Hagen) Willd. - on soil, wood of conifers; 7, 24, 29 [R]. Coll. VR: on wood, 12.08.1917 (H 8004685, sub C. floerkeana).

Cladonia caespiticia (Pers.) Flörke - on soil, bark of Betula spp., Quercus robur, wood of Quercus robur and Picea abies; 4, 5, 8, 20, $23[\mathrm{R}]$.

Cladonia CARIOSA (Ach.) Spreng. - on sandy soil; a4 $\left[R_{1}\right]$.

Cladonia Carneola (Fr.) Fr. - on sandy soil and bark of Pinus mugo; 7, 14, 15, 24 [R].

Cladonia CENOTEA (Ach.) Schaer. - on sandy soil, bark of Alnus glutinosa, Betula spp., Fraxinus excelsior, Picea abies, Pinus sylvestris, wood; 2, 4, 5, 7, 8, 10-13, 19, 20, 22, 23, 25, 27, 28, $32[\mathrm{~F}]$. Coll. VR: on soil and wood, 16-17.06.1938 (Räsänen, 1944; H 8004555).

Cladonia Chlorophaea (Flörke ex Sommerf.) Spreng. s. str. - on soil; $18,24,32$, a30
[R]. Coll. VR: on sandy soil, 17.08.1917 (Räsänen, 1944; H 8004573). Thalli contain fumarprotocetraric acid.

Cladonia CONIOCRAEA (Flörke) Spreng. - on soil, bark of Acer platanoides, Alnus glutinosa, Betula spp., Picea abies, Pinus sylvestris, Quercus robur, wood; 1, 2, 4, 5, 8-13, 19-23, 25-28, 31, 32, 34, a23, a25 [C].

Cladonia CORnuta (L.) Hoffm. subsp. CORnUTA - on soil, bark of Betula spp., Pinus mugo, P. sylvestris, Quercus robur, wood; 2, 3, 5, 7, 11, 15, 19, 23, 24, 29, 32, a11, a18 [O]. Coll. VR: on soil, 1938? (Räsänen, 1944; H 8004603).

Cladonia CRISPATA (Ach.) Flot. var. CRISPATA - on sandy soil and wood of conifers; 3, 7, 15, 19, 24, 32 [R].

Cladonia Cyanipes (Sommerf.) Nyl. - on sandy soil; 7, a30 [R].

Cladonia deformis (L.) Hoffm. - on sandy soil, bark of Pinus mugo, bark and wood of Pinus sylvestris (stump); 7, 15, 24, 32 [R]. Coll. VR: on soil, 17.06.1938 (Räsänen, 1944; H s. n. and 8004434, sub C. arbuscula).

Cladonia digitata (L.) Hoffm. - on soil, bark of Alnus glutinosa, Betula spp., Picea abies, Pinus sylvestris, wood; 2, 5, 8-13, 19-23, 25, 27, 28, 31, 32 [F]. Coll. VR: on soil, 17.06.1938 (Räsänen, 1944; H 8004665).

Cladonia Fimbriata (L.) Fr. - on soil, bark of Pinus sylvestris, Quercus robur, wood; 1-3, 5, 7, 11, 15, 23, 24, 26, 31 [O]. Coll. VR: on soil, 12.08.1917 (H 8004679).

Cladonia floerkeana (Fr.) Flörke - on soil, wood of Pinus sylvestris, plant debris; 7, 24, a4, a30 [R]. Coll. VR: on wood, 12.08.1917 (H 8004685).

Cladonia furcata (Huds.) Schrad. - on soil; 3, 15, 24, a11, a18 [R]. Coll. VR: on soil, 12.08.1917 (Räsänen, 1944; H 8004713).

Cladonia gracilis (L.) Willd. subsp. turbinata (Ach.) Ahti - on soil, bark of Pinus mugo, wood of Pinus sylvestris; 1, 3, 7, 15, 24, 32 [R]. Coll. VR: on mossy soil, 17.06.1938 (Räsänen, 1944; H 8004766).

Cladonia GRAYI G. Merr. ex Sandst. - on soil; 10 $\left[\mathrm{R}_{1}\right]$. Coll. VR: on mossy soil, 17.06.1938 (Räsänen, 1939a, 1944; Hakulinen, 1949; H 8004778; UPS L-74120). Thalli contain grayanic and fumarprotocetraric acids.

Cladonia macilenta Hoffm. - on bark of Pinus mugo, $P$. sylvestris, wood of conifers; 7,10 , 15, 32 [R]. Coll. VR: on wood, 16.06.1938 (Räsänen, 1944; H 8004784).

Cladonia merochlorophaea Asahina - on soil, bark of Pinus mugo; 15, 24, a30 [R]. Coll. 
VR: on mossy soil, 17.06.1938 (H 8004790). Thalli contain merochlorophaeic acid.

i Cladonia norvegica Tønsberg \& Holien - on bark of Alnus glutinosa, Betula spp., Picea abies, rotten wood of Picea abies; 2, 8, 9, 20-22, 28 [O].

Cladonia OCHROCHLORA Flörke - on bark of Populus tremula; $27\left[\mathrm{R}_{1}\right]$.

Cladonia PHYllopHORA Hoffm. - on sandy soil; 3, 7, 24 [R]. Coll. VR: on sandy soil, 17.06.1938 (Räsänen, 1944; H; UPS L-75139).

Cladonia Pleurota (Flörke) Schaer. - on sandy soil; 7, 24, 32 [R]. Coll. VR: on sandy soil, 17.06.1938 (Räsänen, 1944; H 8004817).

Cladonia PyXIDATA (L.) Hoffm. - on sandy soil; 7, 15, 24 [R]. Coll. VR: on soil, 17.06.1938 (Räsänen, 1944; H 8004827).

Cladonia Rangiferina (L.) F. H. Wigg. - on soil, bark of Pinus sylvestris, wood of conifers; $3,7,10,15,19,23,24,29,32$, a11, a18 [O]. Coll. VR: on soil and wood, 16.06.1938 (Räsänen, 1944; H 8004448; UPS L-075419).

Cladonia Rei Schaer. - on soil, bark of Pinus mugo, wood of Pinus sylvestris; 1, 3, 7, 15, 24, a4 [R].

Cladonia squamosa Hoffm. - on sandy soil; a30 $\left[\mathrm{R}_{1}\right]$. Coll. VR: on sandy soil, 17.06.1938 (H 8003576, sub Stereocaulon cumulatum).

Cladonia stellaris (Opiz) Pouzar \& Vězda - on soil; 24, 32, a16, a18 [R]. Coll. VR: on soil and wood, 16-17.06.1938 (Räsänen, 1944; H 8004458, 8004459).

Cladonia stygia (Fr.) Ruoss - on soil; 19, 24, 32 , a30 [R]. Coll. VR: on soil and wood, 16.06.1938 (Ahti \& Hyvönen, 1985; H 8004466; UPS L-075419).

Cladonia subulata (L.) F. H. Wigg. - on soil; 7, 15, 24 [R]. Coll. VR: on soil, 17.06.1938 (Räsänen, 1944; H 8004853).

Cladonia sulphurina (Michx.) Fr. - on soil, bark of Betula spp., Pinus mugo, P. sylvestris, wood of $P$. sylvestris; 1, 2, 7, 10, 11, 15, 19, 32 [O]. Coll. VR: on soil, 17.06.1938 (H).

Cladonia uncialis (L.) F. H. Wigg. subsp. BIUNCIALIS (Hoffm.) M. Choisy - on soil; 24, a16. Subsp. UNCIALIS - on soil; 24, a11, a16, a18, a30. [R].

Cladonia verticillata (Hoffm.) Schaer. - on soil; $3,7,24$ [R].

Cliostomum GRIfFithil (Sm.) Coppins - on bark of Picea abies; a15 [R $]$. Coll. VR: VR4 and without distinct locality, on bark P. abies, 15-16.06.1938 (Räsänen, 1939a, 1940, 1944; Ekman, 1997; H 8004905-8004908, s. n.). s Cliostomum leprosum (Räsänen) Holien \& Tønsberg - on bark of Picea abies; 2, 8, 9, 12, 13, 20, 22, 28, $31[\mathrm{O}]$.

\# Clypeococcum CETRARIAe Hafellner - on thallus of Cetraria islandica on sandy soil; a30 $\left[\mathrm{R}_{1}\right]$.

\# Clypeococcum HYPocenomycis D. Hawksw. - on thalli of Hypocenomyce scalaris on wood and bark of conifers; 10, 28, 32 [R].

Coenogonium Pineti (Ach.) Lücking \& Lumbsch - on bark of Betula spp., Picea abies, wood; 2, 8, 9, 12, 20, 22, 27, 34 [O].

\# Corticifraga fuckeli (Rehm) D. Hawksw. \& R. Sant. - on thallus of Peltigera sp. on sandy soil; a30 $\left[\mathrm{R}_{1}\right]$.

\# Dactylospora homoclinella (Nyl.) Hafellner on thallus of Protoparmeliopsis muralis on granite boulder; $30\left[\mathrm{R}_{1}\right]$.

DiplOsCHISTES SCRUPOSUS (Schreb.) Norman - on granite boulder Kon'-Kamen'; 4 [ $\left.\mathrm{R}_{1}\right]$.

EOPYRENULA LEUCOPLACA (Wallr.) R. C. Harris - on bark of Fraxinus excelsior, a19 $\left[\mathrm{R}_{1}\right]$. Coll. VR: on bark of deciduous tree, 12.08.1917 (H).

EPILICHEN SCABROSUS (Ach.) Clem. - parasite on thallus of Baeomyces rufus on granite (Kon'Kamen'); 4 [ $\left.\mathrm{R}_{1}\right]$.

s Evernia Divaricata (L.) Ach. - on bark of Picea abies; a5 [ $\left.\mathrm{R}_{1}\right]$. Red Data Book of LR (Tzvelev, 2000).

EVERNIA MESOMORPHA Nyl. - on bark of Alnus glutinosa, Betula spp., Picea abies, Pinus mugo, P. sylvestris, wood; 1, 8, 12, 15, 19, 21, 23-25 [O]. Coll. VR: on bark of P. sylvestris and Quercus robur, on wood, 15-16.06.1938 (Räsänen, 1944; Ahlner, 1948; H 8004960; UPS L-85199).

EVERNIA PRUNASTRI (L.) Ach. - on bark of Acer platanoides, Alnus glutinosa, Betula spp., Fraxinus excelsior, Malus domestica, Picea abies, Populus balsamifera, P. tremula, Sorbus aucuparia, Quercus robur, Tilia cordata, wood; 1, 4-6, 10, 17, 18, 23, 25, 26, a23, a29 [O]. Coll. VR: on bark of Acer platanoides, Betula spp., Picea abies, Populus tremula, Quercus robur, Tilia cordata, wood, 12.08.1917, 1938 (Räsänen, 1944; H).

s Felipes leucopellaeus (Ach.) Frisch \& G. Thor on bark of Picea abies; 2, 8, 9, 12, 13, 20-22, $25,27,28,31$, a2 [O].

FrutidELla furfuracea (Anzi) M. Westb. \& M. Svensson - on bark of Pinus mugo; 15 [ $\left.\mathrm{R}_{1}\right]$.

FusCIDEA ARBORICOLA Coppins \& Tønsberg - on bark of Quercus robur, $5\left[\mathrm{R}_{1}\right]$. Thallus contains fumarprotocetraric acid.

Fuscidea PUSILla Tønsberg - on bark of Alnus glutinosa, A. incana, Betula spp., Picea abies, 
Pinus sylvestris, wood of conifers, iron; 1,2 , $8,12,19,20,22-24,26-29,31,32,34[\mathrm{~F}]$. Thalli contain divaricatic acid.

GRAPHIS SCRIPTA (L.) Ach. - on bark of Acer platanoides, Populus tremula, Sorbus aucuparia; 4, 27, 34 [R].

s Gyalecta truncigena (Ach.) Hepp - on bark of Populus tremula; 21, 34 [R].

Gyalolechia flavorubescens (Huds.) Søchting, Frödén \& Arup - on bark of Populus tremula; $17\left[\mathrm{R}_{1}\right]$.

GYROGRAPHA GYROCARPA (Flot.) Ertz \& Tehler - on granite boulder Kon'-Kamen'; 4 [ $\left.\mathrm{R}_{1}\right]$.

\# HaWksworthiana Peltigericola (D. Hawksw.) U. Braun - on thallus of Peltigera sp. on soil; a22 $\left[R_{1}\right]$. - New to WLR, previously known from ELR (Kuznetsova et al., 2012).

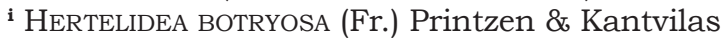
- on standing deadwood of Pinus sylvestris; $10\left[R_{1}\right]$.

\# Heterocephalacria BachmanniI (Diederich \& M. S. Christ.) Millanes \& Wedin - on thallus of Cladonia cornuta on sandy soil; $24\left[\mathrm{R}_{1}\right]$.

\# Heterocephalacria Physciacearum (Diederich) Millanes \& Wedin - on thallus of Physcia aipolia on bark of young Populus tremula; $17\left[\mathrm{R}_{1}\right]$.

\# Homostegia PigGotiI (Berk. \& Broome) P. Karst. on thallus of Parmelia saxatilis on big granite boulder; a2 $1\left[R_{1}\right]$.

HYPOCENOMYCE SCALARIS (Ach.) M. Choisy - on bark of Abies sibirica, Acer platanoides, Betula spp., Picea abies, Pinus sylvestris, Quercus robur, Tilia cordata, wood of conifers; 2, 5, 7, 10-13, 18-21, 23-25, 28, 29, 31, 32, a6 [F]. Coll. VR: on bark of Pinus sylvestris, on wood, 16.06.1938 (Räsänen, 1944; H s. n. and 8005508, sub Imshaugia aleurites).

HYPOGYMNIA FARINACEA Zopf - on granite boulders; $14\left[R_{1}\right]$.

HYPOGYMNIA PHYSODES (L.) Nyl. - on bark of coniferous and deciduous trees, wood, granite boulders and pier, concrete, iron, soil; 1-32, 34, a15, a29, a30 [VC]. Coll. VR: on bark and wood of conifers and Betula spp., 12.08.1917, 1938 (Räsänen, 1944; H; UPS L-86926).

HyPogymnia TUBulosa (Schaer.) Hav. - on bark of coniferous and deciduous trees, wood, granite boulders, iron; $1-6,8-10,12,13,15,16$, 19-23, 26, 27, 29, 31, 32, 34, a29 [C]. Coll. VR: on bark of Picea abies, Quercus robur, on wood, 15-16.06.1938 (Räsänen, 1944; H).

\# Illosporiopsis ChristianseniI (B. L. Brady \& D. Hawksw.) D. Hawksw. - on thallus of Physcia aipolia on bark of Siringa vulgaris; $33\left[\mathrm{R}_{1}\right]$.
\# Illosporium CARneum Fr. - on thallus of Peltigera didactyla on sandy soil; a27 $\left[\mathrm{R}_{1}\right]$.

ImSHAUGia ALEURITES (Ach.) S. L. F. Meyer - on bark of Betula spp., Picea abies, Pinus sylvestris, Ledum palustre, wood of conifers; 10, 19, 24, 32, a6 [R]. Coll. VR: on bark and wood of conifers, 15-16.06.1938 (Räsänen, 1944; H 8005108, 8005508; UPS L-87159).

JAPEWIA SUBAURIFERA Muhr \& Tønsberg - on bark of Betula spp., Picea abies, Pinus mugo, $P$. sylvestris, wood; 2, 8, 12, 13, 15, 19-22, 25, $28,31,34$ [O].

s Lecanactis ABIETina (Ach.) Körb. - on bark of Betula spp., Picea abies, wood; 2, 4, 8, 9, 12, 13, 20-22, 25, 27, 28, 31, a1, a17, a31 [O].

LECANIA CYRTELLA (Ach.) Th. Fr. - on bark of Malus domestica, Populus tremula, Siringa vulgaris; 17, 26, 33 [R]. Coll. VR: on bark of Acer platanoides, 15.06.1938 (H 8005155, sub Lecanora argentata).

LECANIA CYRTELLINA (Nyl.) Sandst. - on bark of Acer platanoides; $4\left[\mathrm{R}_{1}\right]$.

LeCANia NAEGEli (Hepp) Diederich \& van den Boom - on bark of Acer platanoides, Malus domestica, Populus tremula, Siringa vulgaris, Sorbus aucuparia; 4, 5, 16, 17, 26, 33 [R]. Coll. VR: VR2 and without distinct locality, on bark of Salix spp., Sorbus aucuparia, Tilia cordata, 15-16.06.1938 (H 8004231, sub Buellia erubescens; H 8003898, sub Amandinea punctata).

LECANIA SYLVESTRIS (Arnold) Arnold - on concrete, iron; 3, a10 [R].

LECANORA AITEMa (Ach.) Hepp - on bark of Populus balsamifera; $15\left[\mathrm{R}_{1}\right]$.

LECANORA AlBELlula (Nyl.) Th. Fr. var. ALBELlula on bark of Acer platanoides, Quercus robur, wood; 9, 18, 26, 29, a23, a26, a29 [R]. Coll. VR: on wood, 15.06.1938 (H 8004245, sub Buellia schaereri).

LECANORA ALLOPHANA Nyl. - on bark of Populus balsamifera, P. tremula; 6, 17, 18 [R]. Coll. VR: VR2 and without distinct locality, on bark of Acer platanoides, Populus tremula, Sorbus aucuparia, 12.08.1917, 1938 (Räsänen, 1944; H).

LECANORA ANOPTA Nyl. - on wood of conifers; 29 $\left[R_{1}\right]$. - New to WLR, previously known from ELR (Kuznetsova et al., 2007).

LECANORA ARGENTATA (Ach.) Malme (incl. L. subrugosa Nyl.) - on bark of Acer platanoides, Populus balsamifera; 4, 18 [R]. Coll. VR: on bark of Acer platanoides, Sorbus aucuparia and on timber, 15.06.1938 (Räsänen, 1944; H 8005154, 8005155, 8005158). 
LeCANORA CADUbriae (A. Massal.) Hedl. - on bark of Picea abies, bark and wood of Pinus sylvestris; 19, a6, a13 [R].

LECANORA CARPINEA (L.) Vain. - on bark of Acer platanoides, Alnus incana, Malus domestica, Populus balsamifera, P. tremula, Quercus robur, Salix spp., Sorbus aucuparia, wood; 1, 4-6, 16-18, 26, 29, a15 [O]. Coll. VR: VR2, VR3 and without distinct locality, on bark of Acer platanoides, Alnus incana, Fraxinus excelsior, Populus tremula, Quercus robur, Salix spp., Sorbus aucuparia, Tilia cordata, on wood, 12.08.1917, 1938 (Räsänen, 1944; H).

LeCANora CATEILEa (Ach.) A. Massal. - coll. VR: on bark of Acer platanoides, Tilia cordata, 12.08.1917, 1938 (H 8004022, sub Arthonia didyma; $\mathrm{H} 8003983$ and s. n., both sub Alyxoria varia).

LECANORA CENISIA Ach. - on granite boulder; a25 $\left[\mathrm{R}_{1}\right]$. Coll. VR: on wood of Pinus sylvestris, 16.06.1938 (H 8005187, 8005188).

LECANORA CHLAROTERA Nyl. - on bark of Acer platanoides, Alnus glutinosa, Betula spp., Malus domestica, Populus tremula, Quercus robur, Sorbus aucuparia, Tilia cordata, wood; 4, 5, 8, 17, 18, 21, 26, 27, 29, 34, a15, a26 [O]. Coll. VR: on bark of Alnus incana, 16.06.1938 (Räsänen, 1944; H 8004237, sub Buellia erubescens).

LECANORA CIRCUMBOREALIS Brodo \& Vitik. - on bark of Pinus mugo, Ledum palustre, timber fence; 10, 15, a29 [R].

LECANORA EXPALLENS Ach. - on bark of Acer platanoides, Quercus robur, standing deadwood of Pinus sylvestris; 4, 5, 10, 18 [R]. Coll. VR: on bark of Picea abies, 15.06.1938 (Räsänen, 1944; H 8005195). Thalli contain usnic and thiophanic acids, cf. arthothelin, unidentified xanthone "expallens unknown" and zeorin.

LECANORA HYPOPTELLA (Nyl.) Grummann - on bark of Picea abies, bark and wood of Pinus sylvestris; 10, 12, 13, 19, 32 [R].

LECANORA INTRICATA (Ach.) Ach. - on wood of Pinus sylvestris, granite boulders, pebbles and pier, iron; 1, 3, 4, 14, 18, 29, a16 [R]. Coll. VR: on granite and wood, 16-17.06.1938 (Räsänen, 1944; H).

LECANORA MUGHICOLA Nyl. - on timber fence; a29 $\left[R_{1}\right]$. - New to LR. Distribution in Northwestern European Russia outside of LR: Republic of Karelia (Fadeeva et al., 2007). Distribution in Fennoscandia and Baltic countries: Norway, Sweden (Nordin et al., 2011). Ligni- colous species with uneven, continuous, \pm rimose-cracked yellowish-green thallus and innate becoming sessile, round to irregular(angular-) shaped apothecia with margin of same colour with thallus, and red-brown to black disc (Smith et al., 2009).

LECANORA NORVEGICA Tønsberg - on bark of Pinus sylvestris; $11,19[\mathrm{R}]$. Thalli contain atranorin and protocetraric acid.

LECANORA PHAEOSTIGMA (Körb.) Almb. - on bark of Betula sp.; 19 [ $\left.\mathrm{R}_{1}\right]$.

LECANORA POLYTROPA (Ehrh. ex Hoffm.) Rabenh. - on timber and granite piers, granite boulders, pebbles, slate, iron; 1, 3, 4, 14, 16, 29, 30, a16 [O]. Coll. VR: on granite, 16.06.1938 (H 8003835, sub Verrucaria sp.).

LECANORA POPUlicola (DC.) Duby - on bark of Populus tremula; 6, 16, a15 [R].

LECANORA PULICARIS (Pers.) Ach. - on bark of Alnus glutinosa, A. incana, Betula spp., Picea abies, Pinus mugo, Populus balsamifera, Quercus robur, Ledum palustre, timber pier; 1, 5, 10, 15, 19, 20, 23, 29 [O]. Coll. VR: on bark of Alnus incana, 16.06.1938 (Räsänen, 1944; H 8004237, sub Buellia erubescens).

LECANORA SALIGNA (Schrad.) Zahlbr. - on timber fence; a29 $\left[R_{1}\right]$. Coll. VR: VR3, on wood, 15.06.1938 (Räsänen, 1944; H 8005222).

LECANORA SUBinTRICATA (Nyl.) Th. Fr. - on timber fence and pier; 26, 29, a29 [R].

LECANORA SYMMICTA (Ach.) Ach. - on bark of Acer platanoides, Alnus glutinosa, A. incana, Malus domestica, Pinus sylvestris, Populus balsamifera, P. tremula, Quercus robur, Salix spp., Siringa vulgaris, Sorbus aucuparia, Tilia cordata, wood; 1, 3-7, 10, 15-18, 21, 26, 29, 33, a15, a29 [F]. Coll. VR: VR3 and without distinct locality, on bark of Alnus incana, Picea abies, Quercus robur, on wood, 15-16.06.1938 (Räsänen, 1944; H).

LECANORA UMBRINA (Ach.) A. Massal. - on bark of Fraxinus excelsior, Malus domestica, Populus tremula, Quercus robur, Salix spp., Siringa vulgaris, Sorbus aucuparia, wood, granite boulders; 3, 5, 6, 16, 26, 29, 33, 34, a15, a19, a26 [O].

LECANORA VARIA (Hoffm.) Ach. - on timber fence and pier; 29, a29 [R].

LeCidEA ALBOFUSCESCENS Nyl. - on bark of Acer platanoides; 4 [R1].

LECIDEA ERYTHROPHAEa Flörke ex Sommerf. - on bark of Acer platanoides, Populus tremula; $2,4,17,21$ [R].

Lecidea lapicida (Ach.) Ach. var. PANTHERINA Ach. - on granite pebbles; a16 [R1]. 
LECIDEA LEPRARIOIDES Tønsberg - on bark and wood of Picea abies; 2, 9, 12, 13, 20, 22, 25, 28, 31 [O]. Coll. VR: on bark of $P$. abies, 15.06.1938 (H 8005195, sub Lecanora expallens).

LECIDEA NYLANDERI (Anzi) Th. Fr. - on bark of Betula spp., Picea abies, Pinus mugo, P. sylvestris, wood of conifers; 10, 11, 13, 15, 19, 22-25, 32, a1 [O]. Coll. VR: on bark of Picea abies, 15.06.1938 (H 8004995, sub Evernia prunastri).

LECIDEA PLEBEJA Nyl. - on bark of Pinus sylvestris; a30 [R1].

LECIDEA TURGIDUla Fr. - on bark of Picea abies, Pinus sylvestris, wood of conifers; 2, 10, 11, $13,19,31[\mathrm{R}]$.

LeCidella ELAEOCHRoma (Ach.) M. Choisy - on bark of Populus tremula; 6, 12, 16, 34 [R].

LECIDELLA EUPHOREA (Flörke) Hertel - on bark of Populus tremula, timber pier; 6, 17, 27, 29 [R]. Coll. VR: on timber of Pinus sylvestris (wooden pier), 16.06.1938 (Räsänen, 1944; H 8005282).

LECIDELLA FLAVOSOREDIATA (Vězda) Hertel \& Leuckert - on bark of Populus tremula; 6 [R1]. Thallus contains granulosin and arthothelin.

Lecidella stigmatea (Ach.) Hertel \& Leuckert - on concrete; 3 [R1].

LEPRA ALBESCENS (Huds.) Hafellner var. ALBESCENS - on bark of Acer platanoides; 4, a26 [R]. Coll. VR: on bark of Acer platanoides, 15.06.1938 (Räsänen, 1944; H 8000666, 8000667).

LEPRA AMARA (Ach.) Hafellner - on bark of Acer platanoides, Alnus glutinosa, Fraxinus excelsior, Populus tremula, Quercus robur, Tilia cordata; 2, 4-6, 12, 18, 21, 27, 34, a26 [O]. Coll. VR: on bark of Acer platanoides, Picea abies and Sorbus aucuparia, 12.08.1917, 1938 (Räsänen, 1944; H).

LEPRARIA EBURNEA J. R. Laundon - on bark of Alnus glutinosa; 21 [R1]. Thallus contains alectorialic acid.

LEPRARIA ELOBATA Tønsberg - on bark of Betula spp., Picea abies, Tilia cordata; 8, 18, 20, 34 [R]. Thalli contain atranorin, zeorin and stictic acid complex.

LEPRARIA INCANA (L.) Ach. - on bark of Alnus glutinosa, Betula spp., Fraxinus excelsior, Picea abies, Pinus sylvestris, Populus tremula, Quercus robur, Tilia cordata, wood, granite boulder (Kon'-Kamen'); 2, 4, 5, 8-10, 12, $18,20-23,25,27,28,31,32,34$, a $15[\mathrm{~F}]$. Thalli contain divaricatic acid and zeorin, occasionally atranorin.
LEPRARIA JACKII Tønsberg - on bark of Betula spp., Picea abies, Pinus sylvestris, wood; 10, 12, 13, 19, 22, 23 [R]. Coll. VR: on mosses, 16.06.1938 (H s. n.). Thalli contain jackinic/ rangiformic, norjackinic/norrangiformic and roccellic acids.

LEPRARIA LOBIFICANS Nyl. - on bark of Acer platanoides, Alnus glutinosa, Betula spp., Picea abies, Populus tremula, wood of Picea abies, granite boulder; 2, 8, 12, 20-22, 27, 28, 34, a15 [O]. Coll. VR: on mosses, 16.06.1938 (H 8005291 and $\mathrm{H}$ s. n.). Thalli contain atranorin, zeorin and stictic acid complex.

LEPRARIA NEGLECTA (Nyl.) Lettau - on granite boulders, mossy boulders; 1 , a25 [R]. Thalli contain alectorialic acid.

LEPRARIA RIGIDULA (de Lesd.) Tønsberg - coll. VR: on mosses, 16.06.1938 (H 8005293). Thallus contains atranorin and nephrosteranic acid. - New to WLR, previously known from ELR (Kuznetsova et al., 2007).

i Leptogium SATURninum (Dicks.) Nyl. - on bark of Acer platanoides, Populus tremula; 21, a23 [R].

LEPTORHAPHIS ATOMARIA (Ach.) Szatala - on bark of Populus tremula; 2, 6, 16, 17 [R]. Coll. VR: on bark of Populus tremula 16.06.1938 (H 8005497, sub Physcia stellaris).

+ LEPTORHAPHIS EPIDERMIDIS (Ach.) Th. Fr. - on bark of Betula spp.; 1, 5, 23 [R].

\# LicEa PARAsitica (Zukal) G. W. Martin - on thallus of Phaeophyscia orbicularis on bark of Acer platanoides; 18 [R1].

\# Lichenoconium lecanorae (Jaap) D. Hawksw. on apothecia of Lecanora symmicta on bark of Alnus incana and Sorbus aucuparia; 1, $5[\mathrm{R}]$.

\# Lichenoconium pyxidatae (Oudem.) Petr. \& Syd. - on thallus of Cladonia chlorophaea s. 1. on sandy soil; a30 [R1]. - New to WLR, previously known from ELR (Himelbrant et al., 2017).

\# Lichenoconium usneae (Anzi) D. Hawksw. - on thallus of Hypogymnia physodes on bark of Picea abies; 8 [R1].

\# Lichenoconium Xanthoriae M. S. Christ. - on apothecia of Polycauliona polycarpa on bark of Salix sp. and apothecia of Cetraria sepincola on bark of Populus tremula; 16, 17 [R].

\# Lichenodiplis lecanorae (Vouaux) Dyko \& D. Hawksw. - on apothecia of Athallia pyracea on bark of Populus tremula; a15 [R1].

LicheNOMPHALIA UMBELLIFERA (L.: Fr.) Redhead et al. - on mossy rotten deadwood, plant debris; 19, 32, a3 [R]. 
\# Lichenostigma MAURERI Hafellner - on thalli of Bryoria sp. and Usnea hirta on twigs of Picea abies; 13, a5 [R]. - New to WLR, previously known from ELR (Kuznetsova et al., 2012).

s LOBARIA PULMONARIA (L.) Hoffm. - on bark of Acer platanoides, Populus tremula; 21, a14, a23 [R]. Red Data Book of Russian Federation (2008) and LR (Tzvelev, 2000).

LOXOSPORA ELATINA (Ach.) A. Massal. - on bark of Alnus glutinosa, Betula spp., Picea abies, Pinus sylvestris; 2, 8, 10, 13, 19, 20, 27, 28 [O]. Thalli contain thamnolic and elatinic acids.

Melanelia stygia (L.) Essl. - on granite boulder; 1 [R1]. Red Data Book of LR (Tzvelev, 2000).

Melanelixia Glabratula (Lamy) Sandler \& Arup - on bark of Acer platanoides, Fraxinus excelsior, Populus tremula, Quercus robur, Tilia cordata, granite boulder; 4-6, 18, a19 [R]. Coll. VR: on bark of Quercus robur and Tilia cordata, 15-16.06.1938 (Räsänen, 1944; H 8005375).

${ }^{i}$ Melanelixia subargentifera (Nyl.) O. Blanco et al. - on bark of Acer platanoides, Fraxinus excelsior, Quercus robur, 18, a19 [R].

MelaneliXia subaurifera (Nyl.) O. Blanco et al. - on bark of Acer platanoides, Quercus robur, timber fence, granite boulder; 18, a29 [R]. Coll. VR: on bark of Acer platanoides, Quercus robur, Salix sp., 15-16.06.1938 (Räsänen, 1944; H).

Melanohalea EXASPERATA (De Not.) O. Blanco et al. - on bark of Acer platanoides, Populus tremula, Salix spp., timber fence; 5, 6, 16, 17, 21, a15, a29 [R]. Coll. VR: on bark of Fraxinus excelsior, Quercus robur, Salix spp., Tilia cordata, 12.08.1917, 1938 (Räsänen, 1944; H).

Melanohalea EXasperatula (Nyl.) O. Blanco et al. - on bark of Acer platanoides, Fraxinus excelsior, Malus domestica, Populus tremula, Quercus robur, Siringa vulgaris, Sorbus aucuparia, Tilia cordata, wood of conifers, iron; 1 , 3, 5, 6, 18, 21, 26, 29, 33, a19 [O]. Coll. VR: VR3 and without distinct locality, on bark of Acer platanoides, Fraxinus excelsior, Populus tremula, Quercus robur, Sorbus aucuparia, Tilia cordata, wood of conifers, 12.08.1917, 1938 (Räsänen, 1944; H).

Melanohalea olivacea (L.) O. Blanco et al. - on bark of Acer platanoides, Alnus glutinosa, A. incana, Betula spp., Fraxinus excelsior, Picea abies, Pinus sylvestris, Populus balsamifera, P. tremula, Quercus robur, Salix spp., Tilia cordata, wood, granite boulder, iron; 1,
3-5, 14-17, 20, 21, 23, 27, 29, 30, 32, a29 [F]. Coll. VR: on bark of Acer platanoides, Alnus incana, Betula sp., Quercus robur, 12.08.1917, 1938 (Räsänen, 1944; H).

Melanohalea SEPTENTRIONALis (Lynge) O. Blanco et al. - on bark of Populus tremula, timber fence; 17, a29 [R].

Micarea byssacea (Th. Fr.) Czarnota et al. - on rotten deadwood of Picea abies; 28 [R1]. Thallus contains metoxymicareic acid.

MicAREA CONTEXTA Hedl. - on rotten deadwood of conifers; 9, 10, 19, 20, 28 [R]. Thalli contain no lichen substances. - New to LR. Distribution in Northwestern European Russia outside of LR: Republic of Karelia (Fadeeva et al., 2007). Distribution in Fennoscandia and Baltic countries: Norway, Sweden (Nordin et al., 2011). Characterized by inconspicuous immersed thallus with 'micareoid' photobiont 4-7 $\mu \mathrm{m}$ diam., black globose to tuberculate apothecia with purple-violet, $\mathrm{K}^{+}$ green pigment in hymenium (in granules) and hypothecium (intermixed), 1-septate ascospores. Pycnidia immersed to sessile, black; conidia of two types (micro- and mesoconidia) are known (Smith et al., 2009).

Micarea Denigrata (Fr.) Hedl. - on bark of Alnus glutinosa, Picea abies, Pinus mugo, $P$. sylvestris, timber fence; 13, 15, 19, 21, 29, 31, a29 [R]. Contains gyrophoric acid.

MiCAREA ELACHISTA (Körb.) Coppins \& R. Sant. - on standing deadwood of Pinus sylvestris and on upturned roots of Picea abies; 10, a12 [R]. No lichen substances detected by TLC.

MicAREA ERRATICA (Körb.) Hertel, Rambold \& Pietschm. - on granite boulder; 3 [R1].

MicAREA HedLundil Coppins - on bark of Betula spp., Picea abies, rotten deadwood of Picea abies; 2, 8, 20, 22, 25, 28 [R]. No lichen substances detected by TLC. - New to WLR, previously known from ELR (Stepanchikova et al., 2011a).

Micarea MELAena (Nyl.) Hedl. - on bark of Betula spp., Picea abies, Pinus sylvestris, wood of conifers; 2, 8-13, 19-21, 25, 27, 28, 31, 32, $34[\mathrm{~F}]$.

MicAREA MicRococca (Körb.) Gams ex Coppins on bark and wood of Picea abies; 2, 8 [R]. Contains metoxymicareic acid.

Micarea Misella (Nyl.) Hedl. - on wood; 22, 26, a29 [R]. Coll. VR: on wood, 16.06.1938 (H 8005508, sub Imshaugia aleurites).

MiCAREA PELIOCARPA (Anzi) Coppins \& R. Sant. - on bark of Betula spp., Pinus sylvestris, wood; 10, 19, 20 [R]. Contains gyrophoric acid. 
MicAREA PRASINA Fr. s. str. - on bark of Picea abies; 13 [R1]. Contains micareic acid.

s+ Microcalicium AHLNERI Tibell - on standing deadwood of Pinus sylvestris; 10 [R1].

+ Microcalicium arenarium (Hampe ex A. Massal.) Tibell - on upturned roots of Picea sp.; 2 [R1].

i\# Microcalicium disseminatum (Ach.) Vain. on bark and wood of Picea abies, thalli of Chaenotheca spp. on bark of Picea abies; 2, 9, 13, 25, 31 [R]. Coll. VR: VR3, on wood, 15.06.1938 (H 8004398, sub Chaenotheca trichialis).

\# Monodictys ePILePRARIa Kukwa \& Diederich - on thalli of Lepraria incana on bark of Betula sp. and Picea abies; 2, 8 [R]. - New to WLR, previously known from ELR and SPb (Stepanchikova et al., 2011a; Himelbrant et al., 2013).

MontANElia SOREdiata (Ach.) Divakar et al. - on granite boulders, pebbles and granite pier; 1, 3, 18, 29, 30, a16 [R]. Red Data Book of LR (Tzvelev, 2000).

\# Muellerella lichenicola (Sommerf.: Fr.) D. Hawksw. - on apothecia of Athallia pyracea on bark of Populus tremula; 6 [R1]. - New to Northwestern European Russia. Nearest locality in European Russia is known in Murmansk Region (Urbanavichus et al., 2008). Distribution in Fennoscandia and Baltic countries: Norway, Sweden, Finland (Nordin et al., 2011), Estonia (Randlane et al., 2016), Lithuania (Motiejūnaite, 2017). Characterized by usually immersed, small, 70-150 $\mu \mathrm{m}$ diam. perithecia, multispored (ca. 100 per ascus) asci and one-septate, pale- to medium brown ascospores, measuring 4.5-7 × 2-3.5 $\mu \mathrm{m}$ (Triebel \& Kainz 2004). In our specimens, perithecia were $90-100$ $\mu \mathrm{m}$ diam., ascospores were 5-6.5 $\times 2-3 \mu \mathrm{m}$.

MycoBILIMBIA CARNEOAlBIDA (Müll. Arg.) S. Ekman $\&$ Printzen - on bark of Fraxinus excelsior, Populus tremula, mosses on bark of Populus tremula; 2, 4, 12, 21, 34 [R].

MycoBILIMBIA EPIXANTHOIDES (Nyl.) Vitik. et al. - on mosses on bark of Populus tremula; 21, 22 [R].

Mycoblastus alpinus (Fr.) Th. Fr. ex Hellb. - on bark of Picea abies; 13, 28 [R]. Thalli contain atranorin, chloratranorin, planaic and usnic acids.

Mycoblastus SANGUinariUs (L.) Norman - on bark of Betula spp., Picea abies, Pinus sylvestris, wood of $P$. sylvestris; 10, 19, 20, 21, a1 [R]. Coll. VR: on lichens over granite boulder in forest, 17.06.1938 (Räsänen, 1944; H 8000278).
Myriolecis AGARDHIANA (Ach.) Śliwa et al. subsp. AGARDHIANA - On concrete; 3 [R1].

Myriolecis Dispersa (Pers.) Śliwa et al. - on concrete; 3 [R1].

Myriolecis HAGENII (Ach.) Śliwa et al. - on bark of Malus domestica, Populus tremula, Siringa vulgaris; 6, 26, 33, 34 [R]. Coll. VR: on bark of Acer platanoides, Populus tremula, Salix spp., on wood, 15.06.1938 (Räsänen, 1944; H 8003965).

MyRiolecis SAMBUCi (Pers.) Clem. - coll. VR: on bark of Acer platanoides, 15.06.1938 $(\mathrm{H}$ 8005159, sub Lecanora argentata).

Myriolecis SEMIPALlida (H. Magn.) Śliwa et al. - on concrete; 3 [R1].

+ MYcocalicium SubTile (Pers.) Szatala - on wood; $5,10,12,19,23,26$, a6, a20, a29 [R].

NAETROCYMBE PUNCTIFORMIS (Pers.) R. C. Harris - on bark of Populus tremula, Salix spp., Sorbus aucuparia; 16, 27, a15 [R]. Coll. VR: on bark of Acer platanoides, 15.06.1938 (H 8005389, sub Melanohalea olivacea).

NAETROCYMBE RHYPONTA (Ach.) R. C. Harris - coll. VR: on bark of Salix sp., 15.06.1938 (H 8005500, sub Physcia stellaris).

s NEPHROMA PARILE (Ach.) Ach. - on granite boulder in shaded wet place; 34 [R1].

OCHROLECHIA ALBOFLAVESCENS (Wulfen) Zahlbr. on wood of conifers; 20, 31, 32 [R]. Thalli contain variolaric, lichesterinic, protolichesterinic acids and substances 'microstictoides unknowns' (see Kukwa, 2011).

OCHROLECHIA BAHUSIENSIS H. Magn. - on wood of Quercus robur, 5 [R1]. Thallus contains lecanoric, gyrophoric acids and murolic acid complex.

OCHROLECHIA MAHLUENSIS Räsänen - on bark of Betula spp., Picea abies, wood of Picea abies branches; 13, 19, 25, 28, 31 [R]. Thalli contain lecanoric and gyrophoric acids.

OCHROLECHIA MICROSTICTOIDES Räsänen - on bark of Betula spp., Picea abies, Pinus sylvestris, wood of conifers; 9, 10, 12, 13, 19, 20, 22, 25, 27, 28, 31, a2 [O]. Coll. VR: on bark of Picea abies and Pinus sylvestris, 15-16.06.1938 (Räsänen, 1944; Kukwa, 2011; H 80004068000408).

Opegrapha vUlgata (Ach.) Ach. - on bark of Populus tremula, Sorbus aucuparia; 27, a15 [R].

PACHYPHIALE FAGICOLA (Hepp) Zwackh - on bark of Acer platanoides, Malus domestica, Siringa vulgaris, Sorbus aucuparia; 5, 18, 26, 33, a23 [R]. Coll. VR: on bark of Tilia cordata, 16.06.1938 (H 8003898, sub Amandinea punctata). 
PARmelia ERnstiae Feuerer \& A. Thell - on bark of Populus tremula; 6 [R1].

PARMELIA FRAUDANS (Nyl.) Nyl. - coll. VR: on granite boulder, 17.06.1938 (H 8000433). Red Data Book of LR (Tzvelev, 2000).

PARMELIA SAXATILIS (L.) Ach. - on granite boulders; 4, 30, 32, a21, a25 [R]. Coll. VR: on granite boulders, 12.08.1917, 16.06.1938 (Räsänen, 1944; H 8000450, 8000451).

PARMELIA SUlCATA Taylor - on bark of coniferous and deciduous trees, wood, granite boulders and pier, concrete, iron; $1,3-8,12,13$, 15-21, 23, 26, 27, 29, 30, 33, 34, a15, a19, a23, a29 [C]. Coll. VR: VR2, VR3 and without distinct locality, on bark of coniferous and deciduous trees, on wood, 12.08.1917, 1938 (Räsänen, 1944; H).

PARMEliopsis Ambigua (Wulfen) Nyl. - on bark of coniferous and deciduous trees, Ledum palustre, wood, granite boulder; 1, 2, 5-8, 10-13, 15, 18-29, 31, 32, a6, a29 [C]. Coll. VR: on bark of Picea abies, Pinus sylvestris, on wood, 12.08.1917, 1938 (Räsänen, 1944; $\mathrm{H})$.

PARMELIOPSIS HYPEROPTA (Ach.) Arnold - on bark of Betula spp., Fraxinus excelsior, Picea abies, Pinus mugo, P. sylvestris, Populus balsamifera, wood, granite boulder; 5, 7, 8, 12, 13, 15, 19, 23, 28, 32 [O]. On bark of Pinus sylvestris (Räsänen, 1944).

Peltigera APhthosa (L.) Willd. - on soil; 32, a16, a30 [R]. Coll. VR: on soil, 17.06.1938 (Räsänen, 1944; H 8000522, 8000523).

Peltigera Canina (L.) Willd. - on soil, mossy boulders and logs; 1, 3, 7, 18, a24 [R].

Peltigera didactyla (With.) J. R. Laundon - on soil; a22, a27, a30 [R].

Peltigera extenuata (Nyl. ex Vain.) Lojka - on soil; 3, 7, 15, a27 [R].

Peltigera leucophlebia (Nyl.) Gyeln. - on soil; a30 [R1]. Coll. VR: on soil, 17.06.1938 (Räsänen, 1944; H 8000591).

Peltigera Malacea (Ach.) Funck - on soil; a30 [R1].

Peltigera membranacea (Ach.) Nyl. - on mossy logs; a24, a28 [R].

Peltigera neopolydactyla (Gyeln.) Gyeln. - on mossy logs and soil; a7, a24 [R].

Peltigera POLYDACTYlon (Neck.) Hoffm. - on mossy logs; a24 [R1].

Peltigera PRAETEXTATA (Flörke ex Sommerf.) Zopf - on bark of Acer platanoides, on bark and mosses on bark of Populus tremula, wood of Picea abies (log) and granite boulder; 2, 4, 21, 34 [R].
Peltigera Rufescens (Weiss) Humb. - on soil; 3, $15[R]$.

+ Peridiothelia fuliguncta (Norman) D. Hawksw. - on bark of Acer platanoides, Malus domestica; 18, 26 [R].

PERTUSARIA COCCODES (Ach.) Nyl. var. COCCODES on bark of Acer platanoides, Populus tremula, Quercus robur, Tilia cordata; 5, 18, a15, a19, a26 [R]. Coll. VR: on bark of Acer platanoides and Picea abies, 15.06.1938 (Räsänen, 1944; H 8000698, 8000699). Thalli contain norstictic and traces of connorstictic acids.

+ Phaeocalicium polyporaeum (Nyl.) Tibell - on fruit bodies of Trichatum biforme (Fr.) Ryvarden on trunks of Betula sp.; 2, a14 [R].

PhAEOPHYSCIA CILIATA (Hoffm.) Moberg - on bark of Populus tremula; 6, 16, 17, 27 [R].

PhAEOPHYSCIA NIGRICANs (Flörke) Moberg - on bark of Malus domestica, Populus tremula, wood, concrete, iron; 3, 6, 26 [R]. Coll. VR: on bark of Acer platanoides, 12.08.1917, 15.06.1938 (H 8000699, sub Phaeophyscia orbicularis; H 8003791, sub Xanthomendoza fulva).

PHAEOPHYSCIA ORBICULARIS (Neck.) Moberg - on bark of Malus domestica, Populus balsamifera, P. tremula, Quercus robur, Siringa vulgaris, Sorbus aucuparia, concrete; 3, 5, 6, 16-18, 26, 33, a26 [O]. Coll. VR: on bark of Acer platanoides, Populus tremula, 12.08.1917, 15.06.1938 (Räsänen, 1944; H).

PHAEOPHYSCIA SCIASTRA (Ach.) Moberg - on timber and granite piers, concrete; 3, 29 [R]. Coll. VR: on bark of Acer platanoides, on wood, 15-16.06.1938 (Räsänen, 1944; H 8000733; H 8003791, sub Xanthomendoza fulva).

\# Phaeopyxis punctum (A. Massal.) Rambold, Triebel \& Coppins - on thallus of Cladonia coniocraea on lignum of Picea sp.; 2 [R1]. New to WLR, previously known from ELR (Himelbrant et al., 2013).

Phlyctis ARgena (Spreng.) Flot. - on bark of Acer platanoides, Fraxinus excelsior, Malus domestica, Populus balsamifera, P. tremula, Quercus robur, Sorbus aucuparia, Tilia cordata; 2, 4-6, 12, 18, 21, 22, 26, 27, 34, a19, a23 [O]. Coll. VR: on bark of Sorbus aucuparia, 16.06.1938 (H 8004097, 8004099, both sub Bacidia laurocerasi).

Physcia Adscendens H. Olivier - on bark of Acer platanoides, Betula spp., Fraxinus excelsior, Malus domestica, Populus balsamifera, P. tremula, Quercus robur, Siringa vulgaris, Sorbus aucuparia, Tilia cordata, granite boulder, concrete; 3, 5, 6, 17, 18, 26, 33, a26 [O]. 
Coll. VR: on bark of Acer platanoides, Populus tremula, Quercus robur, Tilia cordata, 16.06.1938 (H 8000748).

PHYSCIA AIPOLIA (Ehrh. ex Humb.) Fürnr. - on bark of Acer platanoides, Fraxinus excelsior, Malus domestica, Populus balsamifera, P. tremula, Quercus robur, Salix spp., Siringa vulgaris, Sorbus aucuparia, granite boulder, concrete; 3, 5, 6, 16-18, 26, 27, 33, 34, a15 [O]. Coll. VR: VR3 and without distinct locality, on bark of Acer platanoides, Fraxinus excelsior, Populus tremula, Quercus robur, Tilia cordata, 15-16.06.1938 (H 8000786, 8000787).

Physcia ALNOPHILA (Vain.) Loht. et al. - on bark of Betula spp., Populus tremula, Siringa vulgaris, Sorbus aucuparia; 5, 6, 16, 17, 33, a15 [R].

Physcia CAESIA (Hoffm.) Fürnr. - on bark of Populus tremula, wood of conifers, granite boulders and pier, concrete, slate, iron; 1, 3, 6, 18, 29, 30 [R]. Coll. VR: on bark of Acer platanoides, wood and granite, 15-16.06.1938 (Räsänen, 1944; H 8000796-8000798).

PHYSCIA DUBIA (Hoffm.) Lettau - on bark of Siringa vulgaris, granite boulders and pier, concrete, slate, iron; 1, 3, 29, 30, 33 [R]. Coll. VR: on bark of Sorbus aucuparia, Tilia cordata, wood of conifers, 15-16.06.1938 (H).

PhYSCIA STELlaRIS (L.) Nyl. - on bark of Populus tremula, Quercus robur, Siringa vulgaris; 5, 16, 33 [R]. Coll. VR: on bark of Populus tremula and Salix sp., 12.08.1917, 15.06.1938 (Räsänen, 1944; H 8005497, 8005500).

Physcia TEnella (Scop.) DC. - on bark of Acer platanoides, Malus domestica, Populus balsamifera, P. tremula, Quercus robur, Sorbus aucuparia, granite boulders on the shore, iron; 1, 5, 6, 16-18, 26 [O]. Coll. VR: on bark of Acer platanoides, Quercus robur, Sorbus aucuparia, Tilia cordata, 15-16.06.1938 (Räsänen, 1944; H).

PhysConia DETERSA (Nyl.) Poelt - on bark of Acer platanoides, Populus balsamifera, Quercus robur, Tilia cordata; 18, a19 [R].

PHYSCONIA DISTORTA (With.) J. R. Laundon - on bark of Acer platanoides, Fraxinus excelsior, Populus tremula, Quercus robur, Sorbus aucuparia; 5, 6, 17, 18, 34 [R]. Coll. VR: VR3 and without distinct locality, on bark of Acer platanoides, Fraxinus excelsior, Populus tremula, Quercus robur, Tilia cordata, 12.08.1917, 1938 (Räsänen, 1944; H).

Physconia ENTEROXANTHA (Nyl.) Poelt - on bark of Acer platanoides, Betula spp., Malus domes- tica, Siringa vulgaris, Tilia cordata; 5, 18, 26, 33, a23 [R].

PiCCOLIA OCHROPHORA (Nyl.) Hafellner - on bark of Malus domestica, Sorbus aucuparia; 5, $26[R]$.

Placynthiella dasaea (Stirt.) Tønsberg - on soil, plant debris, wood, bark of Betula spp., Picea abies; 2, 8, 12, 13, 19, 21-23, 26, 29, 32, 34, a16, a29 [O].

Placynthiella icmalea (Ach.) Coppins \& P. James - on soil, plant debris, wood, mossy boulders, bark of Picea abies; 1-3, 5, 7-9, 20, 22, 23, 26, 29, a16 [O]. Coll. VR: on wood, 12.08.1917 (H 8004685, sub Cladonia floerkeana).

Placynthiella Oligotropha (J. R. Laundon) Coppins \& P. James - on sandy soil; 7, 15, 24, a16 [R].

Placynthiella uliginosa (Schrad.) Coppins \& P. James - on soil, plant debris, wood; 3, 5, 7, 8, 14, 15, 24, 26, 29, a8, a16 [O].

Platismatia glauca (L.) W. L. Culb. \& C. F. Culb. - on bark of Acer platanoides, Betula spp., Picea abies, Pinus mugo, P. sylvestris, Quercus robur, wood, granite boulders, iron, soil; 1 , $2,4,5,7-10,12-15,18-25,27,28,31,32$, 34 [C]. Coll. VR: VR4 and without distinct locality, on bark of Picea abies and on wood, 12.08.1917, 1938 (Räsänen, 1944; H).

Pleurosticta aceTabulum (Neck.) Elix \& Lumbsch - on bark of Tilia cordata; 18 [R1]. Red Data Book of LR (Tzvelev, 2000).

Polycauliona candelaria (L.) Frödén, Arup \& Søchting - on bark of Acer platanoides, Quercus robur; a26 [R1]. Coll. VR: on bark of Acer platanoides, Populus tremula, 12.08.1917, 15.08.1938 (H 8003983, sub Alyxoria varia; $\mathrm{H}$ 8005144, sub Lecanora allophana).

PolyCAUliona POLYCARPa (Hoffm.) Frödén, Arup \& Søchting - on bark of deciduous trees, wood, granite boulders; 1, 5, 16-18, 26, 33, a15, a26, a29 [O]. Coll. VR: VR3 and without distinct locality, on bark of Populus tremula, Quercus robur, Tilia cordata and wood, 12.08.1917, 1938 (Räsänen, 1944; H).

\# Polycoccum Pulvinatum (Eitner) R. Sant. - on thallus of Physcia caesia on old granitic pier; 29 [R1].

PoRPIDIA CRUSTUlata (Ach.) Hertel \& Knoph - on iron; a10 [R1].

PorPIDIA MACROCARPA (DC.) Hertel \& A. J. Schwab - on granite boulder; 4. Coll. VR: on granite, 16-17.06.1938 (Räsänen, 1944; H); f. NIGRO- 
CRUENTA (Anzi) Fryday - on granite boulder; a16. [R]. The latter form is characterized by having $\mathrm{K}+$ crimson pigment in exciple (Fryday, 2005); sometimes it is accepted as a separate species Porpidia nigrocruenta (Anzi) Diederich \& Sérus. (e. g. Hafellner \& Türk, 2016).

PorPidia TUBERCUlOSA (Sm.) Hertel \& Knoph - on granite boulder Kon'-Kamen' and pebbles; 4, a16 [R]. Thalli contain confluentic acid, 2'-Omethylperlatolic, 2'-O-methylmicrophyllinic acids.

\# Pronectria leptaleae (J. Steiner) Lowen - on apothecia of Physcia alnophila and P. aipolia on branches of Siringa vulgaris and bark of Populus tremula; 6, 33 [R].

PROTOPARMELIA BADIA (Hoffm.) Hafellner - on granite pebbles; a16 [R1].

PROTOPARMEliopsis MURAlis (Schreb.) M. Choisy - on granite boulders, pebbles and pier, on concrete, slate; 1, 3, 18, 29, 30 [R].

PSEUDEVERNIA FURFURACEA (L.) Zopf - on soil, bark of Acer platanoides, Alnus incana, Betula spp., Picea abies, Pinus mugo, P. sylvestris, Populus balsamifera, Quercus robur, Tilia cordata, wood, iron; 1, 2, 4, 5, 7-10, 13, 15, 18-20, 22-25, 29, 32, a29, a30 [F]. Coll. VR: on bark of Betula sp., Pinus sylvestris, Quercus robur, on wood, 12.08.1917, 1938 (Räsänen, 1944; H).

Pseudoschismatomma Rufescens (Pers.) Ertz \& Tehler - on bark of Sorbus aucuparia; 5 [R1]. Coll. VR: on bark of Picea abies, 15.06.1938 (H 8004908, sub Cliostomum griffithii).

Pseudothelomma ocellatum (Körb.) M. Prieto \& Wedin - on timber fence; a29 [R1].

Psilolechia ClavUlifera (Nyl.) Coppins - on granite boulder under upturned spruce roots; a12 [R1].

Psilolechia Lucida (Ach.) M. Choisy - on bark of Pinus sylvestris, on granite boulder under upturned spruce roots; a12, a15, a30 [R].

PYCNORA SOROPHORA (Vain.) Hafellner - on bark of Pinus sylvestris and wood of conifers; 19, 29, a6 [R].

\# Pyrenochaeta Xanthoriae Diederich - on apothecia and thallus of Xanthoria parietina on bark of Populus tremula; 6 [R1]. - New to LR. Distribution in Northwestern European Russia outside of LR: Republic of Karelia (Fadeeva et al., 2007). Distribution in Fennoscandia and Baltic countries: Estonia (Randlane et al., 2016), Latvia (Motiejūnaite et al., 2016), Lithuania (Motiejūnaitè, 2017).
Characterized by setose pycnidia, elongated, filiform, septate conidiophores, enteroblastic conidiogenous cells appearing as very short lateral branches immediately below each septum of the conidiophores and simple, hyaline conidia 3-4 × 1.4-2 $\mu \mathrm{m}$ (Diederich, 1990). Our specimen was in concurrence with the description in the protologue.

s RAMALINA BALTICA Lettau - on bark of Acer platanoides, Fraxinus excelsior, Populus tremula, Quercus robur, 4, 5, 18, a15, a19, a26 [R]. Coll. VR: VR3 and without distinct locality, on bark of Acer platanoides, Fraxinus excelsior, Picea abies, Populus tremula, Quercus robur, Tilia cordata and on wood, 15.06.1938 (H 8003321-8003325, s. n.). Red Data Book of LR (Tzvelev, 2000).

Ramalina Dilacerata (Hoffm.) Hoffm. - coll. VR: on bark of Picea abies, Populus tremula, Quercus robur, on wood of Pinus sylvestris, 12.08.1917, 1938 (Räsänen, 1944; Ahlner, 1948; H 8003326, 8003327; H 8004099, sub Bacidia laurocerasi). Red Data Book of LR (Tzvelev, 2000).

RAMALINA FARINACEA (L.) Ach. - on bark of Acer platanoides, Betula spp., Fraxinus excelsior, Picea abies, Populus balsamifera, P. tremula, Quercus robur, Sorbus aucuparia, Tilia cordata, wood; 4-6, 17, 18, 21, 26, 34, a23, a26 [O]. Coll. VR: on bark of Acer platanoides, Picea abies, Populus tremula, Quercus robur, Sorbus aucuparia, Tilia cordata, on wood, 12.08.1917, 1938 (Räsänen, 1944; H).

RAMALINA FRAXINEA (L.) Ach. - on bark of Acer platanoides, Fraxinus excelsior, Populus tremula; 6, 12, 18, a19, a26 [R]. Coll. VR: on bark of Acer platanoides, Fraxinus excelsior and Populus sp., 15-16.06.1938 (Räsänen, 1939a, 1944; H 8003410-8003413). Red Data Book of LR (Tzvelev, 2000).

RAMALINA POLLINARIa (Westr.) Ach. - on bark of Acer platanoides, Populus balsamifera, P. tremula, Quercus robur, 5, 6, 18, a19 [R]. Coll. VR: VR3 and without distinct locality, on bark of Acer platanoides, Picea abies, Populus tremula, Quercus robur, Tilia cordata, on wood, 12.08.1917, 1938 (Räsänen, 1944; H).

RAMALINA SINENSIS Jatta - on bark of Sorbus aucuparia; 5 [R1]. Coll. VR: VR3 and without distinct locality, on bark of Acer platanoides, Fraxinus excelsior, Picea abies, Populus tremula, Quercus robur, on wood, 12.08.1917, 1938 (Räsänen, 1944; H 8003443-8003447).

s RAMALINA THRAUSTA (Ach.) Nyl. - coll. VR: VR1, on bark of Picea abies, 15.06.1938 (Räsänen, 
1944; H 8003460, 8003461). Red Data Book of LR (Tzvelev, 2000).

RAMBOLDIA CINNABARINA (Sommerf.) Kalb, Lumbsch \& Elix - on bark of Pinus mugo; 15 [R1].

RAMONIA HIMELBRANTII Gagarina - on wood of conifers; 19, $20[\mathrm{R}]$.

RHIZOCARPON BADIOATRUM (Flörke ex Spreng.) Th. Fr. - on granite boulders and pebbles; 30, a16 [R]. Coll. VR: on granite, 17.06.1938 $(\mathrm{H}$ 8003466).

RHIZOCARPON CINEREOVIRENS (Müll. Arg.) Vain. - on granite boulders on the shore; 1 [R1].

RHIZOCARPON DISPORUM (Nägeli ex Hepp) Müll. Arg. - on old granite pier; 29 [R1]. - New to LR. Distribution in North-Western European Russia outside of LR: Republic of Karelia (Fadeeva et al., 2007). Distribution in Fennoscandia and Baltic countries: Norway, Sweden, Finland (Nordin et al., 2011), Estonia (Randlane et al., 2016), Latvia (Ābolina et al., 2015). Characterized by grey to grey-brown areolate thallus, red-brown $(\mathrm{K}+\mathrm{red})$ epihymenium, one-spored asci and muriform, brown spores (Foucard, 2001).

RHIZOCARPON DISTINCTUM Th. Fr. - on granite boulders and pebbles; 14, a16 [R].

RHIZOCARPON GRANDE (Flörke ex Flot.) Arnold - on granite boulders and pier; 29, 30 [R].

RHIZOCARPON INTERSITUM Arnold - on granite boulders and pebbles; 30, a16 [R].

RHIZOCARPON LAVATUM (Fr.) Hazsl. - on granite boulders and pebbles; 1, 30, a16 [R]. Coll. VR: on granite, 16-17.06.1938 (Räsänen, 1944; H).

RHIZOCARPON MACROSPORUM Räsänen - on granite boulders and old pier; 14, 29, a16 [R].

RHIZOCARPON POLYCARPUM (Hepp) Th. Fr. - on granite boulders and pebbles; 3, a16 [R]. Coll. VR: on granite, 16.06.1938 (Räsänen, 1944; H s. n.).

RINODINA ARCHAEA (Ach.) Arnold - on timber of pier (Räsänen, 1944; Magnusson, 1947).

RINODINA CONRADII Körb. - coll. VR: on timber of pier, 16.06.1938 ( $\mathrm{H}$ 8003504: det. A. H. Magnusson, 1947). - New to WLR, previously known from ELR (Kuznetsova et al., 2007).

RiNodina EXIGUA (Ach.) Gray - on bark of Populus tremula; 17 [R1]. Coll. VR: on timber of pier, 16.06.1938 (H 8003504, sub Rinodina conradii).

RINODINA PYRINA (Ach.) Arnold - coll. VR: on bark of Populus tremula, 12.08.1917 (Räsänen, 1944; Magnusson, 1947; H 8005497, sub Physcia stellaris).
RINODINA SEPTENTRIONALIS Malme - on bark of Acer platanoides, Malus domestica, Pinus mugo, Populus tremula, Sorbus aucuparia, wood; 4, 5, 15-18, 26, 29, a29 [O]. Coll. VR: on bark of Acer platanoides, Salix sp. and on wood, 15-16.06.1938 (H 8003507, 8005359 sub Melanohalea exasperatula, 8005500 sub Physcia stellaris).

RINODINA SOPHODES (Ach.) A. Massal. - on bark of Populus tremula, Salix sp.; 16 [R1]. Reported by Räsänen (Räsänen, 1939a).

RINODINA SUBPARIETA (Nyl.) Zahlbr. - on bark of Acer platanoides, Fraxinus excelsior, Quercus robur, 5, 18, a19 [R]. Thalli contain usnic acid, atranorin and zeorin.

RINODINA TURFACEA (Wahlenb.) Körb. var. ECRUSTACEA (Vain.) H. Olivier - coll. VR: on timber of Pinus sylvestris (wooden pier), 16.06.1938 (H 8005282, sub Lecidella euphorea).

ROPALOSPORA VIRIDIS (Tønsberg) Tønsberg - on bark of Acer platanoides, Populus balsamifera; 4, 15 [R].

s Rostania occultata (Bagl.) Otálora et al. - on bark of Acer platanoides; a23 [R1].

SAGEDIA ZONATA Ach. - on granite boulders; 30 [R1]. Coll. VR: on granite and timber pier, 16-17.06.1938 (Räsänen, 1944; H 8004087; H 8000797, sub Physcia caesia).

+ SAREA Difformis (Fr.) Fr. - on resin of Picea abies; 2, 12 [R].

+ Sarea REsinae (Fr.: Fr.) Kuntze - on resin of Picea abies; 2, 9, 12, 13, 19, 22, 28 [O]. Coll. VR: on resin of $P$. abies, 15.06.1938 (Räsänen, 1944; H 8005583).

s Schismatomma PeRICleum (Ach.) Branth \& Rostr. - on bark of Fraxinus excelsior, 4 [R1]. - Rediscovered in WLR after 124 years [specimen collected by A. O. Kihlman (H 8004268)].

Scoliciosporum CHLOROCOCCUM (Graewe ex Stenh.) Vězda - on bark of Acer platanoides, Picea abies, Pinus mugo, P. sylvestris, Populus balsamifera, P. tremula, Quercus robur, Salix spp., Ledum palustre, wood; 4, 5, 8-10, 15-17, 23, 24, 26, 29 [O].

SCOLICIOSPORUM SAROTHAMNI (Vain.) Vězda - on bark of Acer platanoides, Alnus incana, Populus balsamifera, P. tremula, Salix spp., Siringa vulgaris, Tilia cordata, wood, iron; 1 , 4, 6, 10, 15-18, 21, 26, 29, 33, a15, a29 [O]. SCOLICIOSPORUM UMBRINUm (Ach.) Arnold - on granite boulders, iron; 1, 3, 14, 30, a10 [R].

i ScYtinium TERETIUSCULum (Wallr.) Otálora et al. - on bark of Acer platanoides, Malus domestica, Populus tremula; 21, 26, a23 [R]. 
\# Sphaerellothecium Propinquellum (Nyl.) Cl. Roux \& Triebel - coll. VR: on apothecia of Lecanora carpinea on bark of Tilia cordata, 16.06.1938 (Himelbrant et al., 2016; H 6007649).

+ Stenocybe Pullatula (Ach.) Stein - on bark of Alnus glutinosa, A. incana; 1 [R1]. Coll. VR: on bark of Alnus incana, 16.06.1938 (H 8004237, sub Buellia erubescens).

STEREOCAULON ALPINUM Laurer - on soil, granite boulders; 3, 7, 14, 32, a16 [R].

STEREOCAUlON CONDENSATUm Hoffm. - on soil; 7 , a4 [R].

STEREOCAUlon Cumulatum (Sommerf.) Timdal coll. VR: on sandy soil, 17.06.1938 (Räsänen, 1944; H 8003576).

Stereocaulon glareosum (L. I. Savicz) H. Magn. - on soil; a30 [R1].

STEREOCAUlON PASCHALE (L.) Hoffm. - coll. VR: on sandy soil, 17.06.1938 (H 8003576, sub $S$. cumulatum).

Stereocaulon saxatile H. Magn. - on granite boulders; 1 [R1].

Stereocaulon tomentosum Fr. - on soil; 3, 7, a4 [R]. Coll. VR: on sandy soil, 17.06.1938 (Räsänen, 1944; H 8003568).

\# Stigmidium squamariae (B. de Lesd.) Cl. Roux $\&$ Triebel. - on thallus of Protoparmeliopsis muralis on large boulder on the shore (ornitocoprophilous community); 2 [R1]. - New to LR. Distribution in Northwestern European Russia outside of LR: Republic of Karelia (Fadeeva et al., 2007). Distribution in Fennoscandia and Baltic countries: not reported. Characterized by $60-75 \times 40-55 \mu \mathrm{m}$ ascomata immersed in hymenium of the hosts, all elements $\mathrm{BCr}-$, ascomata wall brown above and colourless at the base and ascospores measuring 9-13 × 4-5 $\mu \mathrm{m}$ (Roux \& Triebel, 1994). Our specimen is concurrent with the description in Roux \& Triebel (1994).

STRANGOSPORA DEPLANATA (Almq.) Clauzade \& $\mathrm{Cl}$. Roux - on bark of Tilia cordata; 18 [R1].

STRANGOSPORA MORIFORMIS (Ach.) Stein - on bark and wood of Pinus sylvestris; 7, 24 [R]. Coll. VR: on bark of Betula sp., 1938? (H s. n., sub Tuckermannopsis chlorophylla).

STRANGOSPORA PINICOLA (A. Massal.) Körb. - on wood of conifers; 29 [R1].

\# TAENIOlella BESChiana Diederich - on thallus of Cladonia sp. on soil; a16 [R1].

Thelenella PERTUSARIElla (Nyl.) Vain. - on bark of Acer platanoides; 34 [R1].

THELOCARPON SUPERELlUM Nyl. - on rotten log deadwood of Picea abies; 20 [R1].
Trapelia coarctata (Sm.) M. Choisy - on vertical shaded and wet surface of big boulder; 23 [R1].

TRAPElia CORTicola Coppins et P. James - on bark of Betula sp.; 23 [R1]. Thallus contains gyrophoric (major) and lecanoric acids and a trace of 5-O-methylhiascic acid. - New to Northwestern European Russia. Distribution in Fennoscandia and Baltic countries: Norway, Sweden (Nordin et al., 2011), Lithuania (Motiejūnaitè, 2017). Characterized by \pm immersed, green to greenish brown thallus with greenish, regular punctiform soralia; usually sterile. Morphologically close to Trapeliopsis spp. (comparison with other sterile sorediate lichens see in Czarnota \& Kukwa, 2009).

TRAPElia Placodioides Coppins \& P. James - on vertical shaded and wet surface of big boulder; 23 [R1].

Trapeliopsis fleXuosa (Fr.) Coppins \& P. James - on bark of Betula spp., Pinus mugo, P. sylvestris, Tilia cordata, wood of conifers; 5 , 7, 10, 11, 15, 19, 23, 29, 31, a29 [O]. Coll. VR: on wood, 16.06.1938 (H 8004784, sub Cladonia macilenta).

TRAPELIOPSIS GRANUlOSA (Hoffm.) Lumbsch - on soil, standing deadwood of Pinus sylvestris; 7, 10, 14, 24, a16 [R].

\# Tremella cetraritcola Diederich \& Coppins on thalli of Tuckermannopsis chlorophylla on twigs of Picea abies; 2, 8 [R]. - New to WLR, previously known from ELR (Himelbrant et al., 2017).

\# Tremella hypogymniae Diederich \& M. S. Christ. - on thallus of Hypogymnia physodes on bark of Picea abies; 8 [R1]. - New to WLR, previously known from ELR (Kuznetsova et al., 2016).

\# Tremella lichenicola Diederich - on thalli of Violella fucata on bark and wood of conifers; 19, 20, 25, 27, 28 [R].

TuCKERMANNOPSIS CHLOROPHYLLA (Willd. ex Humb.) Hale - on bark of Acer platanoides, Betula spp., Fraxinus excelsior, Malus domestica, Picea abies, Pinus mugo, P. sylvestris, Quercus robur, Tilia cordata, wood, granite boulder, iron; 1, 2, 4, 5, 8-10, 12, 13, 15, 18-27, 29, 31, 32, a29 [C]. Coll. VR: on bark of Betula sp., on wood, 16.06.1938 (Räsänen, 1939b, 1944; H).

UMBILICARIA DEUSTA (L.) Baumg. - on granite boulders; 1,30 [R].

UsNeA BARBATA (L.) F. H. Wigg. - coll. VR: VR1 and without distinct locality, on bark of Betula 
sp., Picea abies, 15.06.1938 (H, s. n.: det. P. Halonen, 2005).

UsNeA DASOPOGA (Ach.) Nyl. - on bark of Betula spp., Picea abies, Pinus mugo, P. sylvestris, wood of Picea abies; 2, 12, 13, 15, 19, 21, 23, 25, 28, 31, a14 [O]. Coll. VR: on bark of P. abies, 12.08.1917, 1938 (Räsänen, 1944; H, s. n.: det. F. W. Klingstedt, 1960).

USNEA DIPLOTYPUS Vain. - on bark of Picea abies; 4, $23[R]$.

USNEA cf. FULVOREAGENS (Räsänen) Räsänen - coll. VR: on timber, 15.06.1938 (H 8003627: det. P. Halonen, 1998).

UsNeA GLABRESCEnS (Nyl. ex Vain.) Vain. ex Räsänen - coll. VR: on bark of Betula spp., Picea abies, wood, 15.06.1938 (Räsänen, 1944; H 8003634-8003637: det. P. Halonen, 1998). Thalli contain usnic, norstictic, connorstictic, traces of stictic acids.

UsNeA HIRTA (L.) F. H. Wigg. - on bark of Acer platanoides, Betula spp., Fraxinus excelsior, Picea abies, Pinus sylvestris, Populus tremula, Quercus robur, Tilia cordata, Ledum palustre, wood, iron; $1,4,5,8,10,11,13$, 17-19, 23, 25, 26, 29, 32, a5, a29 [F]. Coll. VR: on bark of Acer platanoides, Betula spp., Quercus robur, on wood, 12.08.1917, 1938 (Räsänen, 1944; H).

USNEA LAPPONICA Vain. - coll. VR: on bark of Acer platanoides, Quercus robur, 15.06.1938 (Räsänen, 1944; Halonen et al., 1999; H 8003681, 8003682).

UsNeA SUBFloRidana Stirt. - on bark of Acer platanoides, Betula spp., Picea abies, Quercus robur, wood; 4, 5, 9, 13, 19, 23, a14 [R]. Coll. VR: on bark of Acer platanoides, Betula sp., Picea abies, Quercus robur and wood, 12.08.1917, 1938 (Räsänen, 1944; H).

USNEA WASMUTHI Räsänen - coll. VR: on bark of Quercus robur, on wood, 15-16.06.1938 (Räsänen, 1944; H 8003729, 8003731).

VERRUCARIA MURALIS Ach. - on concrete; 3 [R1]. Det. Ulf Schiefelbein, 2018.

VERRUCARIA UMBRINULA Nyl. - coll. VR: on shoreline stones, 16.06.1938 (Räsänen, 1944; Pykälä et al., 2012; H 8003835, sub Aspicilia cinerea).

Violella FUCATA (Stirt.) T. Sprib. - on bark of Alnus incana, Picea abies, Pinus mugo, P. sylvestris, Ledum palustre, wood of conifers; 1, 9, 10, $13,15,19,20,22,25-28,31,32,34[\mathrm{~F}]$. Thalli contain atranorin, chloratranorin and fumarprotocetraric acid.

\# VouAuxiomyces sanTESSONII D. Hawksw. - on thalli of Platismatia glauca on branches of $\mathrm{Pi}$ - cea abies; 20 [R1]. - New to WLR, previously known from ELR (Kuznetsova et al., 2012).

VUlPICIDA JUNIPERINUS (L.) J.-E. Mattsson \& M. J. Lai - coll. VR: on wood, 16.06.1938 (Räsänen, 1944; H 8003743). Red Data Book of LR (Tzvelev, 2000).

VulPiCIDA PINASTRI (Scop.) J.-E. Mattsson \& M. J. Lai - on bark of Acer platanoides, Alnus glutinosa, Betula spp., Picea abies, Pinus mugo, P. sylvestris, Populus balsamifera, P. tremula, Quercus robur, wood, plant debris, granite boulders, iron; 1-3, 5-13, 15, 18-21, 23-29, 31, 32, a29 [C]. Coll. VR: on bark of Betula sp., Tilia cordata, on granite, 12.08.1917, 16.06.1938 (Räsänen, 1944; H 8003753).

XANTHOMEndoZa FUlVA (Hoffm.) Søchting et al. on bark of Tilia cordata; 18 [R1]. Coll. VR: on bark of Acer platanoides, 15.06.1938 (H 8003791).

XANTHOPARMElia CONSPERSA (Ehrh. ex Ach.) Hale on granite boulders; 30, a11, a25 [R].

Xanthoparmelia stenophylla (Ach.) Ahti \& D. Hawksw. - on granite boulders; 5, 30, a11, a25 [R].

XANTHORIA PARIETINA (L.) Th. Fr. - on bark of deciduous trees, concrete, iron; 3, 5, 6, 16-18, 26, 27, 33, 34, a15 [O]. Coll. VR: on bark of Acer platanoides, Fraxinus excelsior, Populus tremula, Salix sp., 12.08.1917, 1938 (Räsänen, 1944; H).

\# XanthoriIcola PHYsciae (Kalchbr.) D. Hawksw. on apothecia of Xanthoria parietina on bark of Quercus robur, 5 [R1].

XYLOGRAPHA DIFFORMIS (Vain.) Vain. - on wood; 29 [R1]. Thallus contains norstictic acid. - New to LR. Distribution in Northwestern European Russia outside of LR: Republic of Karelia (Spribille et al., 2014). Distribution in Fennoscandia and Baltic countries: Finland (Nordin et al., 2011). Characterized by ascomata with lateral growth of parallela-type up to $1.2 \mathrm{~mm}$ long, with flexuose margin when young, thallus immersed to superficial, containing norstictic acid (Spribille et al., 2014).

Xylographa PARAllela (Ach.: Fr.) Fr. - on wood; 29 [R1]. Coll. VR: on wood, 16.06.1938 (Räsänen, 1944; H 8003833).

XYLOGRAPHA VITILIGO (Ach.) J. R. Laundon - coll. VR: on wood, 16.06.1938 (H 8005187, sub Lecanora cenisia).

XYLOPSORA CARADOCENSIS (Nyl.) Bendiksby \& Timdal - on bark of Quercus robur, on wood; 5, $7,31[R]$.

XYlopsoRA FRIESII (Ach.) Bendiksby \& Timdal on bark of Picea abies, Pinus sylvestris, on 
wood; 2, 8-10, 12, 13, 19, 20, 22, 25, 31, 32 , a2 [O].

\section{Taxa excluded from lichen flora of Konevets Island:}

Dendrographa latebrarum (Ach.) Ertz \& Tehler - reported by Räsänen (1944) as Crocynia latebrarum (Ach.) Vain., the specimens refer to Lepraria jackii (H s. n.), L. lobificans (H s. n.) and L. rigidula ( $\mathrm{H} 8005293)$.

Lepraria membranacea (Dicks.) Vain. - reported by Räsänen (1944) as Crocynia membranacea (Dicks.) Vain., the specimen refers to L. lobificans (H 8005291).

Physcia tribacia (Ach.) Nyl. - reported by Räsänen (1944), the specimen refers to $P$. tenella (H 8005517).

Ramalina obtusata (Arnold) Bitter - reported by Räsänen (1939a, 1944), the specimens refer to $R$. baltica (H 8003322-8003325, s. n.).

Xanthomendoza fallax (Hepp) Søchting et al. - reported by Räsänen (1944) as Xanthoria substellaris (Ach.) Vain., the specimen refers to Xanthomendoza fulva (H 8003791).

\section{DISCUSSION}

The lichen flora of Konevets Island is surprisingly rich, with a total of 435 species (378 lichens, 46 lichenicolous fungi and 11 non-lichenized saprobic fungi), most of which have been recorded recently (404 species: 351 lichens, 42 lichenicolous fungi and 11 saprobic fungi). These numbers are very high, taking into account the relatively small size of the island and its relief, especially the lack of rocky outcrops. To compare, the list of the whole Berezovye archipelago in Gulf of Finland comprises 356 species (Stepanchikova et al., 2011b), and Tuters Island (also in Gulf of Finland) only 331 species, in spite of presence of rocky communities (Stepanchikova et al., 2017). Even the list of lichens known from the rocky Valaam Archipelago in the northern part of Lake Ladoga is shorter - 363 species (Stepanchikova, Himelbrant, 2004), although it comprises about 70 islands. However, the lichen flora of the Valaam Archipelago is relatively understudied, despite a long history of investigations.

Of the species reported from Konevets in the present paper, one (Acremonium hypholomatis) is new for Russia, three (Caloplaca soralifera, Trapelia corticola, and Muellerella lichenicola) for Northwestern European Russia, seven for LR (Bacidia vermifera, Lecanora mughicola, Micarea contexta, Pyrenochaeta xanthoriae, Rhizocarpon disporum, Stigmidium squamariae, Xylographa difformis), and 16 are for the first time reported from WLR.

The majority of the species recorded in this investigation occur rarely on the island [R] (327 species, $80.9 \%), 136$ of which were recorded only once $\left[R_{1}\right]$, while 54 species $(13.4 \%$ of the lichen flora) were occasional $[\mathrm{O}], 14$ species $(3.5 \%)$ frequent, $[\mathrm{F}], 8$ species $(2.0 \%)$ common $[\mathrm{C}]$, (Bryoria fuscescens, Cladonia coniocraea, Hypogymnia tubulosa, Parmelia sulcata, Parmeliopsis ambigua, Platismatia glauca, Tuckermannopsis chlorophylla, Vulpicida pinastri), and one species (Hypogymnia physodes) very common [VC].

Of the 178 species known from Räsänen's collections and publications all but 31 were also found on Konevets in 2017. Of those not retrieved, five were confined to an old wooden pier (completely destroyed, nowadays only remains of a newer one are present): Biatora beckhausii, Rinodina archaea, $R$. conradii, $R$. turfacea, probably Xylographa vitiligo. Several crustose species, such as the corticolous Alyxoria culmigena, Athallia cerinella, Lecanora cateilea, Myriolecis sambuci, Naetrocymbe rhyponta, Rinodina pyrina and the saxicolous Aspicilia karelica, Verrucaria umbrinula, as well as several Usnea spp. and lichenicolous fungi, might have been overlooked during our recent investigation because of their small size, unremarkable habitus and/or rarity. More noteworthy is the disappearance of some terricolous macrolichens (Cladonia amaurocraea, Stereocaulon cumulatum and S. paschale), which might be connected with increased recreation press, especially along the shores. Nor did we find two conspicuous lichens, the corticolous Vulpicida juniperinus and the saxicolous Parmelia fraudans, both rare in Leningrad Region and regionally red-listed due to decrease in localities during the last 100 years. Another significant species, Ramalina thrausta, was collected by Räsänen from an old spruce forest near Kon'-Kamen'. The species disappeared from Konevets not just by accident, but because of some serious changes in the forest community. In the Soviet period an electrical line was built 
not far from Kon'-Kamen', which changed the microclimate in the forest. The water disappeared from the Holy spring nearby the boulder and some old spruces died and were cut down in the $20^{\text {th }}$ century (Anna Voskresenskaya, pers. comm.). Lepraria rigidula and most probably Ramalina dilacerata had previuously been found at the same locality, but also these species have disappeared. $R$. thrausta is a critically endangered species in Leningrad Region, exclusively confined to old-growth undisturbed wet spruce forests (Andersson et al., 2009). Nowadays it is only known from the eastern part of the region. The last record from the mainland part of Karelian Isthmus dates back to $1941(\mathrm{H})$.

The contemporary lichen flora of Konevets Island comprises 404 species, most of which are corticolous (232 species, $57.4 \%$ of modern diversity) and lignicolous lichens (136 species, $33.7 \%)$. The lichen communities on stones $(85$ species, $21.0 \%$ ) and soil (61 species, $15.1 \%$ ) are comparatively poor. Some species were also recorded on iron (27 species), mosses (10 species), epiphytic algae (4 species), fruit bodies of polypores (2 species), and resin of conifers (2 species). A rather high number of lichenicolous species were registered (39 species, 9.7\% of lichen flora), which in our opinion, indicates that the lichen diversity of the island nowadays is comparatively well studied.

One of the most significant parts of Konevets lichen flora is found in spruce forests, in which 188 species were recorded $(46.7 \%$ of the lichen flora), of these species 56 were found in spruce forests only. Compared to the mainland part of Karelian Isthmus, the spruce forests of Konevets are well-preserved and little disturbed. The average age of the spruces is 180 years and more, and there are no traces of fires or clear cuts in the majority of these communities. According to our data, the old-growth spruce forests on Konevets Island cover an area of c. $2.2 \mathrm{~km}^{2}$ (almost a quarter of the total area of the island) and are mainly found in the northern part of the island. Here we recorded 11 habitat specialists of oldgrowth biologically valuable forests (Andersson et al., 2009): Biatoridium monasteriense, Chaenotheca chlorella, Chaenothecopsis viridireagens, Cliostomum leprosum, Evernia divaricata, Felipes leucopellaeus, Gyalecta truncigena, Lecanactis abietina, Lobaria pulmonaria, Ramalina baltica,
Schismatomma pericleum, in addition to 13 indicator species: Alectoria sarmentosa, Arthonia spadicea, Bacidia fraxinea, B. polychroa, $B$. rubella, Chaenotheca brachypoda, C. stemonea, Chaenothecopsis consociata, C. nigra, Cladonia norvegica, Leptogium saturninum, Microcalicium disseminatum, Scytinium teretiusculum. In the mainland part of Karelian Isthmus the last records of Alectoria sarmentosa date to 1913 $(\mathrm{H})$, of Chaenothecopsis viridireagens to 1924 (HFR), of Evernia divaricata to 1941 (H), and of Schismatomma pericleum to 1893. The nearest modern locality within Karelian Isthmus for Biatoridium monasteriense and Ramalina baltica is Maly Berezovy Island in Gulf of Finland (Alexeeva \& Himelbrant, 2007). Felipes leucopellaeus and Lecanactis abietina are not known from other localities in Karelian Isthmus. Hence the lichen diversity of Konevets spruce forests is unique for the northwestern part of Leningrad Region and therefore deserves special protection.

Pine forests, including swampy habitats, hosted 148 species $(36.7 \%)$. In the southern part of the island pine forests are selectively cut. Most important findings were made in old-growth swampy pine forests in the northernmost part of the island, such as the habitat specialists of biologically valuable forests (Andersson et el., 2009) Buellia arnoldii and Microcalicium ahlneri, and the indicator species Calicium denigratum, Carbonicola anthracophila, and Hertelidea botryosa. Of these Buellia arnoldii was last recorded in Karelian Isthmus in $1935(\mathrm{H})$ and Hertelidea botryosa in 1893 (H; Vainio, 1934). All reliable records of Carbonicola anthracophila are confined to the eastern part of Leningrad Region.

Other lichenologically rich habitats are the old broadleaved park and the alleys surrounding the monastery. Altogether 144 species (35.7\%) were recorded there, including the habitat specialists Lobaria pulmonaria, Ramalina baltica, and Rostania occultata and the indicator species Arthonia spadicea, A. vinosa, Bacidia fraxinea, B. rubella, Leptogium saturninum, Melanelixia subargentifera, Pertusaria coccodes, and Scytinium teretiusculum (Andersson et al., 2009).

The lichens of other habitats were less diverse: anthropogenic wastelands bore 112 species $(27.8 \%)$, aspen stands 110 species $(27.3 \%)$, open 
shores of Lake Ladoga, including boulders, willow shrubs along the shores and an old port, 97 species $(24.1 \%)$, and grey alder stands 52 species $(12.9 \%)$. Noteworthy was the habitat formed by Pinus mugo and Populus balsamifera on cape Strelka (Hiekkaniemi) in SW part of the island (sample area 15). The trees were planted at the beginning of the $20^{\text {th }}$ century, and the community is now self-renewing and resembles shrub thickets characteristic to mountain regions. In this area we found 49 species, most of which were recorded also in other habitats on the island, except for Frutidella furfuracea, Lecanora aitema and Ramboldia cinnabarina. On the same sandy cape Strelka we found another noteworthy shoreline habitat: wasteland overgrown with $\mathrm{Ni}$ photrichum canescens (Hedw.) Bednarek-Ochyra $\&$ Ochyra and Polytrichum piliferum Hedw. Several lichen species grew among the mosses on the sandy soil, including the usually epiphytic Bryoria fuscescens, Hypogymnia physodes and Platismatia glauca (sample area 14).

'Island specificity' is not high in Konevets lichen flora, but some species occurring there are found in Leningrad Region predominantly along shores, such as Bacidina inundata, Sagedia zonata, Cliostomum griffithii, and Ramalina baltica.

Altogether ten species nowadays known from Konevets Island are included in the Red Data Book of Nature of Leningrad Region (Tzvelev, 2000): Alectoria sarmentosa, Arctoparmelia centrifuga, Bryoria nadvornikiana, Evernia divaricata, Lobaria pulmonaria, Melanelia stygia, Montanelia sorediata, Pleurosticta acetabulum, Ramalina baltica, and $R$. fraxinea. Additionally, 17 species are recommended for inclusion into the new edition of the Red Data Book of Leningrad Region: Acrocordia cavata, Arthonia spadicea, A. vinosa, Bacidia fraxinea, B. polychroa, Biatoridium monasteriense, Buellia arnoldii, Calicium denigratum, Carbonicola anthracophila, Felipes leucopellaeus, Lecanactis abietina, Melanelixia subargentifera, Microcalicium ahlneri, Peltigera membranacea, Pertusaria coccodes, Rostania occultata, and Schismatomma pericleum. In conclusion, Konevets Island now bears a rich and diverse lichen biota, exceptionally wellpreserved in comparison to the mainland part of Karelian Isthmus. There is no doubt that the natural forest communities and historical parks of the island are worthy of protection.
Nowadays most of Konevets Island is under the control of the Konevsky Monastery, which protects it against fires, illegal cuttings and unorganized tourism. It is necessary to continue a responsible management of this area. Especially important is the control of visitors. We also strongly recommend to avoid cuttings in spruce forests of the island.

\section{ACKNOWLEDGEMENTS}

DH, EK and IS would like to thank Hegumen Alexander, head of the Konevsky Monastery, for organization and support of our investigations, as well as Anna Voskresenskaya, Director of the Monastery Museum, the monastery brethren and pilgrimage service for friendly help during the investigation. We are grateful to Teuvo Ahti and other colleagues in the Botanical Museum, University of Helsinki, as well as colleagues in the Museum of Evolution, Uppsala University for their help during our work in herbaria of $\mathrm{H}$ and UPS. Ludmila Konoreva thanks Sergey Chesnokov (Komarov Botanical Institute of Russian Academy of Sciences, St. Petersburg) for the help in chromatography studies of Micarea specimens. We are grateful to Ivan Frolov (Botanical Garden of the Urals Branch of Russian Academy of Sciences, Ekaterinburg) for identification of Caloplaca soralifera, to Ulf Schiefelbein (Rostock, Germany) for identification of Verrucaria muralis, and to Olga V. Gurova (Husum, Germany) for linguistic correction of the manuscript. We express our gratitude to anonymous reviewer and Tiina Randlane for valuable corrections and comments. The study was carried out within the framework of the institutional research project (no. AAAA-A18-118031590042-0) of the Komarov Botanical Institute RAS. The work of IS and Ludmila Konoreva was supported by Russian Foundation for Basic Research (grants 16-04-01488 and 18-34-00332).

\section{REFERENCES}

Āboliṇa, A., Piterāns, A. \& Bambe, B. 2015. Lichens and bryophytes in Latvia. Checklist. Daugavpils, Daugavpils Universitātes Akadēmiskais apgāds "Saule». 213 pp. (In Latvian, English introduction).

Ahlner, S. 1948. Utbredningstyper Bland Nordiska Barrträdslavar. Acta Phytogeographica Suecica 22: 1-257. (In Swedish). 
Ahti, T. \& Hyvönen, S. 1985. Cladina stygia, a common, overlooked species of reindeer lichen. Annales Botanici Fennici 22(3): 223-229.

Alexeeva, N. M. \& Himelbrant, D. E. 2007. Lichens. In: Tzvelev, N. N. (executive ed.) [Volkova, E. A., Glazkova, E. A., Isachenko, G. A. \& Khramtsov, G. A. (eds.)]. Environment and biological diversity of Berezovye Islands Archipelago (The Gulf of Finland). St. Petersburg. Pp. 213-229. (In Russian).

Andersson, L., Alexeeva, N. M. \& Kuznetsova, E. S. (eds). 2009. Survey of biologically valuable forests in North-Western European Russia. Vol. 2. Identification manual of species to be used during survey at stand level. St. Petersburg. 258 pp. (In Russian).

Czarnota, P. \& Kukwa, M. 2009. Contribution to the knowledge of some poorly known lichens in Poland. III. Trapelia corticola and the genus Vezdaea. Folia Cryptogamica Estonica 46: 25-31.

Diederich, P. 1990. New or interesting lichenicolous fungi 1. Species from Luxembourg. Mycotaxon 37: 297-330.

Diederich, P. \& Braun, U. 2009. First lichenicolous record of Acremonium hypholomatis (anamorphic Ascomycota). Bulletin de la Société des naturalistes luxembourgeois 110: 97-99.

Ekman, S. 1997. The genus Cliostomum revisited. Symbolae Botanicae Upsalienses 32: 17-28.

Fadeeva, M. A., Golubkova, N. S., Vitikainen, O. \& Ahti, T. 2007. Conspectus of lichens and lichenicolous fungi of the Republic of Karelia. Petrozavodsk. 194 pp. (In Russian, English summary).

Foucard, T. 2001. Svenska skorplavar och svampar som växer på dem. Stockholm. 392 pp. (In Swedish).

Fryday, A. M. 2005. The genus Porpidia in northern and western Europe, with special emphasis on collections from the British Isles. Lichenologist 37(1): 1-35. https://doi.org/10.1017/ S0024282904014628

Gagarina, L. V. 2015. Gyalectoid lichens (families Gyalectaceaea Stizenb. and Coenogoniaceae (Fr.) Stizenb.) in Extratropical Eurasia. St. Petersburg. 240 pp. (In Russian).

Hafellner, J. \& Türk, R. 2016. Die lichenisierten Pilze Österreichs - eine neue Checkliste der bisher nachgewiesenen Taxa mit Angaben zur Verbreitung und Substratökologie. Stapfia 104(1): 1-216.

Hakulinen, R. 1949. Über das Vorkommen von Cladonia Grayi Merr. im östlichen Fennoskandien. Archivum Botanici Societatis Zoologicae-Botanicae Fennicae "Vanamo" 4(1): 17-20.

Hakulinen, R. 1962. Die Flechtengattung Anaptychia Körb. in Ostfennoskandien. Archivum Botanici Societatis Zoologicae-Botanicae Fennicae "Vanamo" 17(3): 121-133.

Halonen, P., Myllys, L., Ahti, T. \& Petrova, O. V. 1999. The lichen genus Usnea in eastern Fennoscandia. III. The shrubby species. Annales Botanici Fennici 4(36): 235-256.
Himelbrant, D. E., Motiejūnaitė, J., Pykälä, J., Schiefelbein, U. \& Stepanchikova, I. S. 2013. New records of lichens and allied fungi from the Leningrad Region, Russia. IV. Folia Cryptogamica Estonica 50: 23-31. https:/ / doi.org/10.12697/fce.2013.50.04

Himelbrant, D. E., Stepanchikova, I. S., Motiejūnaitè, J., Gagarina, L. V. \& Dyomina, A. V. 2016. New records of lichens and allied fungi from the Leningrad Region, Russia. VII. Folia Cryptogamica Estonica 53: 25-34. https://doi.org/10.12697/ fce. 2016.53 .04

Himelbrant, D. E., Stepanchikova, I. S., Motiejūnaitè, J., Gerasimova, J. V., Kuznetsova, E. S., Dyomina, A. V. \& Tsurykau, A. G. 2017. New records of lichens and allied fungi from the Leningrad Region, Russia. VIII. Folia Cryptogamica Estonica 54: 63-70. DOI: https://doi.org/10.12697/ fce. 2017.54 .11

Konevets Island. The guidebook. 2015. Konevets Island. 152 pp. (In Russian).

Kotiranta, H., Uotila, P., Sulkava, S. \& Peltonen, S.-L. (eds). 1998. Red Data Book of East Fennoscandia. Helsinki. 351 pp.

Kukwa, M. 2011. The lichen genus Ochrolechia in Europe. Gdańsk. 309 pp.

Kuznetsova, E., Ahti, T. \& Himelbrant, D. 2007. Lichens and allied fungi of the Eastern Leningrad Region. Norrlinia 16: 1-62.

Kuznetsova, E. S., Kataeva, O. A., Himelbrant, D. E. \& Motiejūnaitè, J. 2016. Lichens and allied fungi of the Ragusha River Protected Area (Leningrad Region, Russia). Folia Cryptogamica Estonica 53: 71-80. DOI: https://doi.org/10.12697/ fce.2016.53.09

Kuznetsova, E. S., Motiejūnaitè, J., Stepanchikova, I. S., Himelbrant, D. E. \& Czarnota, P. 2012. New records of lichens and allied fungi from the Leningrad Region, Russia. III. Folia Cryptogamica Estonica 49: 31-37.

Magnusson, A. H. 1947. Studies in non-saxicolous species of Rinodina mainly from Europe and Siberia. Meddelanden från Göteborgs Botaniska Trädgård 17: 191-338.

Motiejūnaitè, J., Chesnokov, S. V., Czarnota, P., Gagarina, L. V., Frolov, I., Himelbrant, D., Konoreva, L. A., Kubiak, D., Kukwa, M., Moisejevs, R., Stepanchikova, I., Suija, A., Tagirdzhanova, G., Thell, A. \& Tsurykau, A. 2016. Ninety-one species of lichens and allied fungi new for Latvia with a list of additional records from Kurzeme. Herzogia 29(1): 143-163. https://doi.org/10.13158/ heia.29.1.2016.143

Motiejūnaitè, J. 2017. Supplemented checklist of lichens and allied fungi of Lithuania. Botanica Lithuanica 23(2): 89-106. https://doi.org/10.1515/ botlit-2017-0011

Muchnik, E., Wilk, K., Vondrák, J. \& Frolov, I. 2014. Contribution to the knowledge of the genus Caloplaca in Central European Russia. Polish 
Botanical Journal 59(2): 263-270. https://doi. org/10.2478/pbj-2014-0043

Nordin, A., Moberg, R., Tønsberg, T., Vitikainen, O., Dalsätt, Å., Myrdal, M., Snitting, D. \& Ekman, S. 2011. Santesson's Checklist of Fennoscandian Lichen-forming and Lichenicolous Fungi. Ver. April 29, 2011 - http://130.238.83.220/santesson/ home.php (15 March 2018).

Orange, A., James, P. W. \& White, F. J. 2001. Microchemical methods for the identification of lichens. London. $101 \mathrm{pp}$.

Pykälä, J., Stepanchikova, I. S., Himelbrant, D. E., Kuznetsova, E. S. \& Alexeeva, N. M. 2012. The lichen genera Thelidium and Verrucaria in the Leningrad Region (Russia). Folia Cryptogamica Estonica 49: 45-57.

Randlane, T., Saag, A. \& Suija, A. 2016. Lichenized, lichenicolous and allied fungi of Estonia. Ver. December 31, 2016. http: / / esamba.bo.bg.ut.ee/ checklist/est/home.php (14 March 2018).

Räsänen, V. 1921. Einige neue und bemerkenswerte Flechtenfunde in Finnland. Meddelanden af Societas pro Fauna et Flora Fennica 46: 156-174.

Räsänen, V. 1939a. Die Flechtenflora der nördlichen Küstengegend am Laatokka-See. Annales Botanici Societatis Zoologicae-Botanicae Fennicae "Vanamo" 12(1): 1-240.

Räsänen, V. 1939b. Lichenes Fenniae exsiccati a Museo botanico Universitatis Helsingiensis 8-11 (No. 351-550): 1-50.

Räsänen, V. 1940. Lichenes Fenniae exsiccati a Museo botanico Universitatis Helsingiensis 12-14 (No. 551-700): 1-50.

Räsänen, V. 1944. Eine Pflanzenexkursion zu der Klosterinsel Konevitsa im westlichen Teil des Laatokka-Sees. Annales Botanici Societatis Zoologicae-Botanicae Fennicae "Vanamo" 20(15): 3-64.

Rassadina, K. A. 1930. On the lichens of former Petergof County of Leningrad Province. Proceedings of the Botanical Nuseum of AS USSR 22: 223-271. (In Russian).

Red Data Book of Russian Federation (Plants and Fungi). 2008. Moscow. 855 pp. (In Russian).

Roux, C. \& Triebel, D. 1994. Révision des espèces de Stigmidium et de Sphaerellothecium (champignons lichénicoles non lichénisés, Ascomycetes) correspondant à Pharcidia epicymatia sensu Keissler ou à Stigmidium schaereri auct. Bulletin de la Société Linéenne de Provence 45: 451-542.

Smith, C. W., Aptroot, A., Coppins, B. J., Fletcher, A., Gilbert, O. L., James, P. W. \& Wolseley, P. A. (eds.). 2009. The lichens of Great Britain and Ireland. The British Lichen Society, London. 1046 pp.
Spribille, T., Resl, P., Ahti, T., Pérez-Ortega, S., Tønsberg, T., Mayrhofer, H. \& Lumbsch, H. T. 2014. Molecular systematics of the wood-inhabiting, lichen-forming genus Xylographa (Baeomycetales, Ostropomycetidae) with eight new species. Symbolae Botanicae Upsalienses 37(1): 1-87.

Stepanchikova I. S., Andreev M. P., Himelbrant D. E., Motiejūnaitė J., Schiefelbein U., Konoreva L. A. \& Ahti T. 2017. The lichens of Bolshoy Tuters Island (Tytärsaari), Leningrad Region, Russia. Folia Cryptogamica Estonica 54: 95-116. https:/ / doi.org/10.12697/fce.2017.54.14

Stepanchikova, I. S. \& Himelbrant, D. E. 2004. The history of investigations and contemporary data on lichens of Valaam Archipelago (Karelia ladogensis) / / Proceedings of VIII Conference of Young Botanists in St. Petersburg, 17-21 May 2004. St. Petersburg. P. 87. (In Russian).

Stepanchikova, I. S., Himelbrant, D. E., Kukwa, M. \& Kuznetsova, E. S. 2011a. New records of lichens and allied fungi from the Leningrad Region, Russia II. Folia Cryptogamica Estonica 48: 85-94.

Stepanchikova I. S., Schiefelbein U., Alexeeva N. M., Ahti T., Kukwa M., Himelbrant D. E. \& Pykälä J. 2011 b. Additions to the lichen biota of Berezovye Islands, Leningrad Region, Russia. Folia Cryptogamica Estonica 48: 95-106.

Tibell, L. 1973. Notes on Caliciales. II. Some Species from the Nordic Countries. Svensk Botanisk Tidskrift 67: 445-455.

Titov, A. N. 2006. Mycocalicioid fungi (Mycocaliciales) of Holarctic. KMK, Moscow. 296 pp. (In Russian).

Triebel, D. \& Kainz, C. 2004. Muellerella. In Lichen Flora of the Greater Sonoran Desert Region, Vol. 2 (T. H. Nash III, B. D. Ryan, P. Diederich, C. Gries \& F. Bungartz (eds). Lichens Unlimited, Arizona State University, Tempe, Arizona, pp. 673-674.

Tzvelev, N. N. (ed.). 2000. Red Data Book of Nature of the Leningrad Region. Vol. 2. Plants and Fungi. St. Petersburg. 672 pp. (In Russian).

Urbanavichus, G., Ahti, T. \& Urbanavichene, I. 2008. Catalogue of lichens and allied fungi of Murmansk Region, Russia. Norrlinia 17: 1-80.

Vainio, E. A. 1934. Lichenographia Fennica IV. Lecideales II. Acta Soc. Fauna Fl. Fenn. 57(2-3): 1-539.

Vondrák, J. \& Hrouzek, P. 2006. Caloplaca soralifera, a new species from Europe. Graphis Scripta 18: 6-15. 
Appendix 1. List of sampling locations in Leningrad Region, Priozersk District, Konevets Island (Konevitsa)

No Description, geographical coordinates, biotope

Date

Standard sample areas:

$1 \quad$ NW shore, W of bay Filippova lakhta (Filippuksenlahti), 60 $52^{\prime} 52.4^{\prime \prime} \mathrm{N}, 30^{\circ} 36^{\prime} 41.1^{\prime \prime} \mathrm{E}$, grey alder stand with birch, 26.07 .2017 willow, with mossy boulders and remains of old car, along the shore line

2 N part, SE of bay Filippova lakhta (Filippuksenlahti), 6052'48.3”N, 30³7’03.1”E, old-growth spruce forest (age of 26.07 .2017 spruces 170-200 years) with green mosses, dwarf shrubs and Oxalis acetosella L.

3 E shore, cape Bely Nosok N of bay Valkoinenhiekka, 6052'07.8”N, 30³8'06.1”E, young pine stand on sand, with 26.07 .2017 granite boulders and ruins of military constructions

4 W part, Kon'-Kamen' with chapel of St. Arsenij, 6051'26.1”N, 30³5'47.1”'E, huge glacial boulder with a chapel above 26.07 .2017 it, surrounded by shadowy old-growth spruce forest with broadleaved trees

5 S part, meadow S of Sviataya Hill and Kazansky Skete, 60 51'03.9”'N, 30³5'53.7”E, alley of broadleaved trees (ca. 27.07.2017 150-200 years old)

6 S part, Sviataya Hill, 60 51'12.7”N, 30³5'54.5”E, open old-growth aspen stand with Calamagrostis sp. between a 27.07 .2017 meadow and spruce forest

$7 \quad$ NE part, S of cape Vargosy (Varkaanniemi), 6052'51.6”N, 30³8’03.1”E, anthropogenic wasteland with lichens and 28.07 .2017 graminoids near the shore

$8 \quad$ NE part, between capes Vargosy (Varkaanniemi) and Bely Nosok, 6052'27.3”'N, 30³7'53.1'E, old-growth spruce 28.07.2017 forest (age of spruces 150-170 years) with green mosses, dwarf shrubs, Oxalis acetosella, ferns, Linnaea borealis L. and Maianthemum bifolium (L.) F. W. Schmidt

$9 \quad \mathrm{~N}$ part, S of bay Filippuksenlahti (Filippova lakhta), 60 $52^{\prime} 45.0^{\prime \prime} \mathrm{N}, 30^{\circ} 37^{\prime} 31.0^{\prime \prime} \mathrm{E}$, old-growth spruce forest (age of 28.07 .2017 spruces 160-180 years) with Sphagnum spp. and dwarf shrubs

10 Same place, $60^{\circ} 52^{\prime} 40.4^{\prime \prime} \mathrm{N}, 30^{\circ} 37^{\prime} 33.6^{\prime} \mathrm{E}$, open old-growth pine forest (age of pines 250-300 years) with Ledum palustre 28.07 .2017 L., Empetrum nigrum L., Vaccinium vitis-idaea L., V. myrtillus L. and V. uliginosum L. on a ridge between two swamps

11 N part, NW of cape Bely Nosok, 6052'17.9”'N, 30³7'24.6”'E, secondary pine forest (age of pines 80-100 years) with 28.07 .2017 green mosses and Vaccinium myrtillus

12 N part, N of Zmeinaya Hill, 60 52'18.9”'N, 30³6'39.3”E, old-growth spruce forest (age of spruces ca. 100 years) with 29.07 .2017 green mosses and Vaccinium myrtillus, with many logs and mossy boulders

13 Central part, S of Zmeinaya Hill, 6051'52.9”N, 30³6’35.2”E, old-growth spruce forest (age of spruces more than 29.07 .2017 170 years) with Sphagnum spp. and Vaccinium myrtillus

14 SW shore, cape Strelka (Hiekkaniemi), 6050’45.3”N, 30³4’04.4”E, shoreline Racomitrium-lichen wasteland com- 29.07 .2017 munity with granite boulders on sand

15 same place, 6050'48.4”N, 30³4'12.0”E, Populus balsamifera L.-Pinus mugo Turra community on sandy spit

16 SE part, nameless islet NE of Kamenny Islet (Kivisaari), E of cape Suravuniemi, 6050’28.2”N, 30³7’27.6”E, willow 30.07.2017 shrubs and young aspens on pebble shore with boulders

17 SE part, NE of Konevsky Skete, 6050'29.6”N, 30³6’34.5”E, young aspen stand in a former meadow

18 SW part, SW side of the Monastery, 60 $50^{\prime} 51.9^{\prime \prime} \mathrm{N}, 30^{\circ} 34^{\prime} 55.2^{\prime \prime} \mathrm{E}$, alley and park of broadleaved trees

19 N part, SE of bay Filippova lakhta (Filippuksenlahti), 6052'44.9”N, 30³6'48.8”E, old-growth swampy pine stand 31.07 .2017 (age of pines 200-210 years) with Sphagnum spp., Ledum palustre and Eriophorum vaginatum L.

$20 \mathrm{~N}$ part, SE of bay Filippova lakhta (Filippuksenlahti), 6052’31.2” N, 30³7’01.6”E, spruce forest with green mosses, 31.07 .2017 Vaccinium myrtillus and Oxalis acetosella

$21 \mathrm{~N}$ part, NE of Zmeinaya Hill, 60²'24.8”N, 30³7'11.4”E, old-growth spruce forest (age of spruces more than 18031.07 .2017 years) with birch and aspen, with young rowan and maple undergrowth, and with Vaccinium myrtillus and Hepatica nobilis Schreb.

22 Central part, SW of Zmeinaya Hill, 60 $51^{\prime} 49.6^{\prime \prime} \mathrm{N}, 30^{\circ} 36^{\prime} 14.7^{\prime \prime} \mathrm{E}$, old-growth spruce forest with aspen, birch and pine 01.08 .2017 (age of spruces 100-170 years, aspens - ca. 80 years), with green mosses, horsetails, Vaccinium myrtillus, Calamagrostis sp. and ferns

23 Central part, NE of Kon'-Kamen', 60 $51^{\prime} 40.1^{\prime \prime} \mathrm{N}, 30^{\circ} 36^{\prime} 14.8^{\prime \prime} \mathrm{E}$, birch forest with Sphagnum spp. and Carex sp.

24 E shore, bay Valkoinenhiekka (Valkiahieta), 6051'50.3”N , 30³7’29.2”E, open old-growth pine forest (age of pines 01.08 .2017 more than 180 years) with lichens and mosses on sandy shore

25 E shore, S part of bay Valkoinenhiekka (Valkiahieta), 6051’43.6” N, 30³7’27.6”'E, old-growth spruce forest with green 01.08 .2017 mosses and Vaccinium myrtillus, with young rowans 
26 S part, meadow S of Sviataya Hill and Kazansky Skete, 60 $51^{\prime} 6^{\prime \prime} \mathrm{N}, 30^{\circ} 35^{\prime} 51.8^{\prime \prime} \mathrm{E}$, old apple tree garden (age of some 02.08 .2017 trees up to ca. 180 years) near the meadow

27 E part, W of cape Rodushka (Roduskoj), 6051'16.0”N, 30³7’02.5”E, old-growth spruce forest (age of spruces 140-150 03.08.2017 years) with green mosses, Vaccinium myrtillus and ferns

28 E part, SW of bay Valkoinenhiekka (Valkiahieta), 6051’32.5” N, 30³6’56.3”E, old-growth spruce forest with birch 03.08 .2017 and black alder, with Sphagnum spp., Vaccinium myrtillus and patches of green mosses

29 SW shore, bay Vladychnaya (Konevetskaya), 6050'45.8”N, 30³4'53.2”'E, old harbor with a wooden pier and granite pier 03.08 .2017

30 E shore, unnamed cape S of cape Rodushka (Roduskoj), 6050'54.6” N, 30³7’26.1”'E, large granite boulders on the shore 04.08 .2017

31 E part, SW of cape Rodushka (Roduskoj), 6051'00.5” N, 30³6'58.9”E, old-growth spruce forest with green mosses, 04.08 .2017 Vaccinium myrtillus and patches of Sphagnum spp.

32 S part, NE of Konevsky Skete, 6050'46.6”'N, 30³6’34.3”'E, old-growth pine forest (age of pines 170-220 years) with 04.08 .2017 traces of selective cuttings, with green mosses, lichens and Vaccinium myrtillus, and with mossy boulders

33 SW part, N part of Monastery, household yard, 605 '56.8” N, 30³5’08.6”'E, old lilac shrubs in front of ruins

34 S part, N of Konevsky Skete, 6050'41.2”N, 30³6'15.3”E, aspen forest with spruce, birch, Oxalis acetosella, Vaccinium 05.08 .2017 myrtillus, Rubus saxatilis L., with mossy boulders

Additional plots:

a1 N part, S of bay Filippova lakhta (Filippuksenlahti), 60 $52^{\prime} 43.9^{\prime \prime} \mathrm{N}, 30^{\circ} 37^{\prime} 07.7^{\prime \prime} \mathrm{E}$, old-growth spruce forest (age of 26.07 .2017 spruces 170-200 years) with green mosses, dwarf shrubs and patches of Sphagnum spp.

a2 E part, W of cape Bely Nosok, 60²’08.6”N, 30³7’41.6”E, old-growth spruce forest (age of spruces 170-200 years) 26.07.2017 with green mosses and Vaccinium myrtillus

a3 Central part, W of bay Valkoinenhiekka (Valkiahieta), 6051'42.1”N, 30³6'44.4”E, old wet road in old-growth 26.07 .2017 spruce forest

a4 NE shore, $\mathrm{S}$ of cape Vargosy (Varkaanniemi), 6052'44.3”N, 30³8’03.8”'E, anthropogenic wasteland with mosses, 28.07 .2017 graminoids and separate pines near the shore

a5 N part, S of bay Filippova lakhta (Filippuksenlahti), 60 $52^{\prime} 40.9^{\prime \prime} \mathrm{N}, 30^{\circ} 37^{\prime} 33.8^{\prime \prime} \mathrm{E}$, edge of old-growth spruce forest 28.07 .2017 near swamp with Carex sp.

a6 Same place, 60'52'38.8”N, 30³7’34.9”E, faintly meliorated peatbog with separate old pines and standing deadwood 28.07 .2017

a7 N part, W of Zmeinaya Hill, 60 $52^{\prime} 08.6^{\prime \prime} \mathrm{N}, 30^{\circ} 36^{\prime} 41.3^{\prime \prime} \mathrm{E}$, old road in spruce forest with green mosses and Vaccinium 29.07.2017 myrtillus

a8 SW part, W of Sviataya Hill, 60 $51^{\prime} 11.0^{\prime \prime} \mathrm{N}, 30^{\circ} 35^{\prime} 22.8^{\prime \prime} \mathrm{E}$, road in secondary pine forest

29.07.2017

a) SW part, W of the Monastery, 60'50'54.7”N, 30³4’34.4” E, pine forest with green mosses and Vaccinium myrtillus

a10 SE part, NE of Konevsky Skete, 6050’31.5”N, 30³6’42.4”E, small wasteland with remnants of bus and other vehicles 30.07 .2017 surrounded by secondary spruce forest

a11 SE part, unnamed islet NE of Kamenny Islet (Kivisaari), E of cape Suravuniemi, 6050’25.3”N, 30³7’14.4”E, open 30.07.2017 pine stand on pebbles

a12 N part, SE of bay Filippova lakhta (Filippuksenlahti), 6052'49.9”N, 30³7’01.0”'E, old-growth spruce forest (age of 31.07 .2017 spruces 170-200 years) with green mosses, dwarf shrubs and Oxalis acetosella

a13 Same place, 60'52'41.4”N, 30³6'59.8”'E, pine forest with green mosses and Vaccinium myrtillus

NW part, NW of Zmeinaya Hill, 6052’26.4”N, 30³6’37.4”E, aspen forest with spruces (age of aspens ca. 80 years)

31.07 .2017

a15

W shore, N of Kon'-Kamen', 6052'00.6”'N, 30³5'50.9”E, old aspens and spruces along the road

31.07.2017

a16

W shore, small cape $\mathrm{N}$ of Kon'-Kamen', 60 $51^{\prime} 42.0^{\prime \prime} \mathrm{N}, 30^{\circ} 35^{\prime} 43.1^{\prime \prime} \mathrm{E}$, disturbed pine forest on sandy shore

31.07.2017

a17 E shore, N part of bay Valkoinenhiekka (Valkiahieta), 6052’04.0”N, 30³7’36.2”E, old-growth spruce forest (age of 01.08 .2017 spruces ca. 150 years) with green mosses, Vaccinium myrtillus and patches of Sphagnum spp.

a18 E shore, SW of cape Rodushka (Roduskoj), 6051'13.2”N, 30³7’24.0”E, open pine forest with lichens and mosses 01.08 .2017 on sandy shore

a19 S part, Sviataya Hill, N of Kazansky Skete, 6051'07.3”N, 30³5'55.9”'E, alley of old broadleaved trees

02.08.2017

a20 S part, Sviataya Hill, in front of Kazansky Skete, 6051’07.1”N, 30³5'51.5”E, separate old broadleaved trees

02.08.2017

a21 S part, NE of the Monastery, 60 $511^{\prime} 02.1^{\prime \prime} \mathrm{N}, 30^{\circ} 35^{\prime} 39.3^{\prime \prime} \mathrm{E}$, big boulder in pine forest with green mosses and Vac- 02.08 .2017 cinium myrtillus

a22 S part, E of the Monastery, 60 $50^{\prime} 54.4^{\prime \prime} \mathrm{N}, 30^{\circ} 35^{\prime} 35.3^{\prime \prime} \mathrm{E}$, anthropogenic wasteland (dump) in pine forest with green 02.08 .2017 mosses and Vaccinium myrtillus 
a23 SW part, Monastery, near the wall of an old monk cemetery, 6050'52.5” N, 30³5'10.2”'E, separate old broadleaved trees 02.08 .2017

a24 Central part, W of cape Rodushka (Roduskoj), 6051'22.6”N, 30³6'48.5”E, young birch-aspen stand with spruces, 03.08 .2017 with Convallaria majalis L. and Daphne mezereum L.

a25 Central part, E of Sviataya Hill, 6051'16.4”N, 30³6'35.7”'E, big boulders in a meliorated meadow

a26 SW part, S corner of the Monastery, 60 $50^{\prime} 48.6^{\prime \prime} \mathrm{N}, 30^{\circ} 35^{\prime} 05.3^{\prime \prime} \mathrm{E}$, old broadleaved alley and park

a27 S shore, bay Vladychnaya (Konevetskaya), 6050'45.7”'N, 30³5'26.6”'E, pines between the road and the shore

a28 W part, vicinity of Kon'-Kamen' with chapel of St. Arsenij, 6051'26.1”N, 30³6’02.9”E, open place with mossy logs 04.08 .2017 in secondary spruce forest

a29 SW part, NW of Monastery, 60'50'57.2”N, 30³4'56.8”'E, wooden fence between the pasture and the road

a30 SW shore between Kon'-Kamen' and Riihiranta beach, 6051'05.3-25.1”N, 30³4'46.5”-35'35.0”E, old-growth pine 05.08 .2017 forest and open lichen-graminoid community on a sandy shore

a31 S part, N of Konevsky Skete, 6050'41.4”N, 30³6'16.5”E, old-growth spruce forest with green mosses and Vaccinium 05.08.2017 myrtillus, with logs

Historical localities:

VR1 Pirunkirkka - W part of Konevets Island, Kon'-Kamen' (Pirunkivi, Pirunkirkka) with chapel of St. Arsenij (same with 15.06 .1938 locality 4), [6051'26”N, $30^{\circ} 35^{\prime} 47^{\prime \prime} \mathrm{E}$ ], probably old-growth spruce forest

VR2 Pyhälähde - W part of Konevets Island, N of Svyataya Hill in vicinity of Kon'-Kamen', former place of Holy Spring 15.06 .1938 (same with locality a28), [60 $\left.51^{\prime} 26^{\prime \prime} \mathrm{N}, 30^{\circ} 36^{\prime} 03^{\prime \prime} \mathrm{E}\right]$

VR3 Skiitta - S part, most probably surroundings of Kazansky Skete (close to localities 5, 26, a19 and a20), [6051’N, 16.06.1938 $\left.30^{\circ} 36^{\prime} \mathrm{E}\right]$, probably an alley of broadleaved trees and a wooden building (or fence)

VR4 Valkiahieta (bay Valkoinenhiekka, 'White sands' in English) - vicinities of a large sandy bay on E shore (close to locali- 16.06 .1938 ties 24,25 and a17), [60 $52^{`} \mathrm{~N}, 30^{\circ} 37^{\prime} \mathrm{E}$ ], probably old-growth spruce forest 